\title{
Ciclos económicos y gasto público: un análisis de cambio estructural para la región centro de México, 1980-2015
}

Pablo Mejía Reyes* y Gina Sánchez Peña**

\section{RESUMEN}

En el presente trabajo se analiza la relación entre el ciclo económico y los componentes del gasto público en los estados del centro de México durante el periodo 1980-2015, considerando la posibilidad de cambios estructurales. Se encuentra que una alta proporción de tales cambios ocurre durante la segunda mitad de los años ochenta y el transcurso de los noventa, así como en torno de las recesiones de 2001-2003 y 2008-2009. Las estimaciones para los diferentes regímenes identificados sugieren que la política del gasto público nacional fue pro-cíclica hasta la primera década de este siglo, pero con coeficientes cada vez menores o no significativos. Este patrón se repite en varios rubros del gasto a nivel estatal, aunque los resultados no son siempre robustos. En general, se puede concluir que en los años recientes el gasto público estatal, altamente dependiente de los recursos federales, ha sido inefectivo como instrumento de estabilización o se ha usado con otros fines en los estados analizados.

Palabras clave: ciclos económicos, gasto público, cambios estructurales, región centro, México.

Clasificación JEL: C49, E32, E62.

Profesor-Investigador del Centro de Investigación en Ciencias Económicas (CICE), Facultad de Economía, Universidad Autónoma del Estado de México, México. Correo electrónico: pemejiare@uaemex.mx

** Egresada de la Licenciatura en Economía de la Universidad Autónoma del Estado de México, México. Correo electrónico: gina_sp_2006@hotmail.com 


\section{ABSTRACT \\ Economic cycles and public spending: an analysis of structural change for the central region of Mexico, 1980-2015}

This paper analyzes the relationship between the business cycles and public expenditure components in the states of Central Mexico during the period 1980-2015, considering the possibility of structural changes. It was found that a high proportion of such changes occurs during the second half of the eighties and over the nineties, as well as around the recessions of 2001-2003 and 2008-2009. The estimates for the corresponding identified regimes suggest that the national public expenditure was pro-cyclical until the first decade of this century, but with decreasing or insignificant coefficients. This pattern is also present in several components of expenditure at a state level, although the results are not always robust. In general, it can be concluded that in recent years the public expenditure, highly dependent on federal resources, has been ineffective as a stabilization instrument or has been used for other purposes in the states analyzed in this paper.

Keywords: business cycles, public spending, structural changes, central region, Mexico

JEL CLassification: C49, E32, E62

\section{INTRODUCCIÓN}

Las economías de mercado han experimentado fluctuaciones cíclicas prácticamente desde su surgimiento, y la macroeconomía ha puesto especial énfasis en la determinación de sus causas, mecanismos de transmisión y efectos sobre la estabilidad macroeconómica y el bienestar de las familias (Sorensen y Whitta-Jacobsen, 2010; Knoop, 2015). Por su evidente relevancia, numerosos estudiosos han propuesto una amplia gama de políticas de estabilización para amortiguarlas, asumiendo diferentes circunstancias. Sus propuestas incluyen el diseño de estrategias y el uso de instrumentos monetarios, financieros, cambiarios, arancelarios y fiscales, entre otros (Blanchard et al., 2010; Hori, 2017) que pretenden aminorar las caídas en las fases recesivas y evitar el sobrecalentamiento en las fases de auge.

Aunque persiste el debate teórico, principalmente entre las vertientes modernas de las corrientes clásica y keynesiana, sobre si el 
gobierno debe o no intervenir en la economía, la experiencia reciente de la Gran Recesión ha demostrado que la aplicación de políticas de estabilización pueden ser determinantes en la amortiguación de la caída en la actividad económica y en el retorno a la senda del crecimiento. La profundidad y virulencia de ese episodio ha llevado a la extracción de múltiples lecciones entre las que destacan el convencimiento de que los ciclos económicos no son asunto del pasado ${ }^{1}$ y que el gobierno puede contribuir efectivamente a estabilizar la economía. No sin opiniones contrarias, la evidencia ha mostrado que la política fiscal puede ser efectiva bajo ciertas circunstancias, por lo que la discusión en torno a su uso sistemático ha retornado al centro de la discusión (Esquivel, 2010; Eggertsson, 2011; Alesina, 2012; Aizenman y Jinjarak, 2011).

Desde una perspectiva histórica más amplia, en la literatura se ha documentado que, en general, la política fiscal ha sido contra-cíclica (estabilizadora) $^{2}$ en la gran mayoría de los países desarrollados, lo que contrasta notablemente con lo que ha ocurrido en diversos países en desarrollo, entre los que se cuentan los latinoamericanos, y desde luego México (Burnside y Meshcheryakova, 2005; Reyes y Mejía, 2012). Una política fiscal pro-cíclica como ésta no sólo no abona a la suavización de las fluctuaciones cíclicas de la economía, sino que contribuye a amplificarlas al expandir la demanda durante los periodos de auge, pero sobre todo al reducirla en las fases recesivas (Burnside y Meshcheryakova, 2005; Spiegel, 2007).

Aunque una política fiscal pro-cíclica se ha justificado con base en la disponibilidad de recursos públicos durante las diferentes fases del ciclo económico y la necesidad de enviar señales a los mercados de que se mantendrán los equilibrios macroeconómicos, recientemente ha habido un debate sobre la necesidad de seguir políticas contra-cíclicas en México (Burnside y Meshcheryakova, 2005; Esquivel, 2010). No obstante, esta discusión ha estado completamente ausente a nivel subnacional posiblemente por el escaso margen de maniobra de los

1 Desde finales de la década de los años noventa se venía presentado una ola de profundo optimismo que llevó a bautizar este periodo como la "Gran Moderación", término acuñado por Stock y Watson (2003), para referirse a la reducción de la volatilidad de las variables macroeconómicas de la economía estadounidense.

2 Una variable es contra-cíclica si se mueve en sentido opuesto al comportamiento del indicador del ciclo, y viceversa. Una política fiscal es contra-cíclica si es expansiva durante una recesión, y viceversa. 
gobiernos estatales al ser altamente dependientes de las transferencias del gobierno federal, que en promedio alcanzan el $80 \%$ de sus ingresos (Fonseca et al., 2013). ${ }^{3}$ En esa lógica, el análisis de la política fiscal como instrumento de estabilización en los estados es prácticamente inexistente. Al parecer, el único trabajo que aborda este tema es el de Fonseca et al. (2013), quienes proponen un instrumento de estabilización basado en el manejo de las transferencias federales de modo que las entidades federativas ahorren recursos de las participaciones durante las expansiones con el fin de afrontar los descensos del gasto público en las fases recesivas.

Este planteamiento está en línea con la necesidad de dotar a los gobiernos subnacionales de instrumentos que les permitan contrarrestar las fluctuaciones cíclicas pues hasta ahora el manejo del gasto público ha tenido efectos contrarios en diversas latitudes. Por ejemplo, Miyazaki (2013) y Carmignani y Laurenceson (2013) examinan la relación entre la política fiscal y las fluctuaciones cíclicas de las prefecturas de Japón y las provincias de China, respectivamente. En general, en ambos casos se reporta que la política fiscal (la inversión pública del tipo discrecional en el primer caso) amplifica las fluctuaciones de los ciclos económicos regionales debido a su carácter pro-cíclico. Por su parte, Rodden y Wibbels (2010) examinan la correlación entre las fluctuaciones de la producción regional y las partidas presupuestarias en regresiones de panel para 7 países: Argentina, Australia, Brasil, Canadá, Alemania, India y Estados Unidos. Encuentran que tanto los impuestos de fuentes propias como los gastos de las provincias generalmente son pro-cíclicos, aun cuando en algunos casos el gasto federal sea contracíclico, lo que refleja las limitantes que enfrentan las provincias para lidiar con la volatilidad del ciclo económico derivadas de su limitada autonomía fiscal.

Con el fin de contribuir a sentar las bases para una discusión mejor informada sobre la conveniencia de dotar a los gobiernos subnacionales (estatales) de instrumentos de estabilización, el objetivo del presente

En el federalismo mexicano, los estados y municipios han cedido muchas de sus atribuciones recaudatorias a la federación, la cual, a cambio, transfiere importantes recursos de regreso para que los primeros con sus funciones. No obstante, eso ha significado una alta dependencia histórica de aquellos que no se ha revertido significativamente a pesar de las múltiples reformas a la Ley de Coordinación Fiscal que regula las relaciones entre los tres niveles de gobierno (Velázquez, 2006; Mandujano, 2010; Arechederra y Urzúa, 2017). 
trabajo es analizar la relación entre el ciclo económico y los componentes del gasto público en los estados del centro de México durante el periodo 1980-2015. Dado que durante el periodo analizado se han adoptado diferentes reformas estructurales y políticas de estabilización y se han modificado las leyes que regulan la relación entre los gobiernos estatales y la federación, se considera la posibilidad de cambios estructurales en dicha relación; los resultados se contrastan con la experiencia nacional.

La que consideramos aquí como región centro está conformada por la Ciudad de México (antes Distrito Federal) y los estados de México, Guanajuato, Hidalgo, Morelos, Puebla, Querétaro y Tlaxcala. Su importancia se refleja en el hecho de que en conjunto representa el $6.6 \%$ de la superficie del país, aporta más del 30\% del PIB, alberga al $38 \%$ de la población y capta el $43.9 \%$ del total de inversión extranjera directa. ${ }^{4}$ Llama la atención, sin embargo, que a pesar de su importancia haya recibido tan poca atención en la literatura económica.

El resto de este artículo se estructura en cuatro secciones. En la primera se describe la relación entre gasto público y ciclo económico en las principales teorías modernas para identificar las causas de su posible vinculación. Posteriormente se presenta la metodología para medir el co-movimiento entre tales variables, así como el enfoque econométrico para evaluar si su relación es estable o ha cambiado en el tiempo. En la tercera sección se presentan y discuten los principales resultados. Finalmente, se establecen las conclusiones.

\section{CONSIDERACIONES TEÓRICAS}

Las compras de bienes y servicios del Estado pueden afectar a la actividad económica en el corto plazo de dos formas principales: como un choque de demanda que puede generar fluctuaciones cíclicas en las principales variables macroeconómicas o como un instrumento de estabilización que puede amortiguar su magnitud (Jones, 2014; Sorensen y

4 Los estados considerados para la región centro se obtuvieron de una adaptación de la regionalización que utiliza el Banco de México (Banxico, 2011). La información de superficie, PIB y población se obtuvo del Instituto Nacional de Estadística y Geografía (INEGI) (http://www. inegi.org.mx/) y la de inversión extranjera de la Secretaría de Economía (www.gob.mx/se). 
Whitta-Jacobsen, 2010; Knoop, 2015). Su relevancia ha llevado a las diferentes escuelas de pensamiento a analizar sus efectos, atribuyéndoles distintos grados de importancia en términos de su impacto en las diferentes variables macroeconómicas.

Por un lado, la teoría de los ciclos económicos reales (CER), ${ }^{5}$ propuesta por Kydland y Prescott (1982), postula que en un marco con agentes racionales, mercados competitivos e información simétrica, las fluctuaciones cíclicas de variables como la producción y el empleo corresponden a las respuestas de agentes optimizadores a los choques reales recurrentes y aleatorios que experimenta la economía. En particular, de acuerdo con este enfoque un aumento en el gasto público hace que los trabajadores aumenten su oferta de trabajo para compensar la pérdida de riqueza que provocarán los mayores impuestos esperados para financiar ese gasto. Como respuesta se reduce el consumo presente y se incrementa el esfuerzo de trabajo y, por ende, la producción, aunque a una tasa salarial menor, lo que empeora el bienestar de la población. Entonces, bajo estas consideraciones, la política fiscal podría ser procíclica, aunque implícitamente indeseable por sus efectos nocivos en el bienestar.

Por otro lado, en la década de los años ochenta aparece la nueva economía keynesiana (NEK) como una nueva versión del enfoque keynesiano que adopta fundamentos microeconómicos y expectativas racionales y asume competencia imperfecta, costos de menú y externalidades para fundamentar la existencia de salarios y precios nominales rígidos. En ese marco, en línea con los planteamientos keynesianos tradicionales, considera que los choques de demanda constituye la principal fuente de los ciclos económicos, aunque también aceptan los generados del lado de la oferta. Desde la perspectiva de la $\mathrm{NEK}^{6}$, un incremento temporal del gasto público eleva la demanda de bienes y disminuye el ahorro nacional deseado. A corto plazo, mientras los precios se mantienen inalterados, se genera un aumento tanto en la

Véanse Plosser (1989), Stadler (1994) o Rebelo (2005) para exposiciones panorámicas de este enfoque. En este enfoque se enfatiza el papel de los choques reales, como cambios tecnológicos, alteraciones del gasto público o los impuestos, o modificaciones en las preferencias de los consumidores, en contraposición con los choques nominales que usualmente se asumen neutrales.

6 Véanse Greenwald y Stiglitz (1987), Mankiw (1989) y Stadler (1994), para una visión más detallada. 
tasa de interés real como en la producción. Como las empresas satisfacen el aumento de la demanda al nivel de precios fijo, el empleo también aumenta sin que se altere su oferta si es que existen salarios de eficiencia. ${ }^{7}$ Entonces, el aumento de las compras del Estado sólo afecta a la producción elevando la demanda agregada, por lo que el tipo de política de gasto descrita anteriormente se considera expansiva, y viceversa, al menos en el corto plazo en lo que los precios se ajustan a su nuevo nivel de equilibrio (Mankiw, 1989).

Esta posibilidad da pie a que la política fiscal pueda utilizarse como instrumento de estabilización para contrarrestar la magnitud de las fluctuaciones cíclicas, especialmente durante las recesiones cuando la eficacia de la política monetaria es limitada y el recorte de los salarios puede no ser efectivo ante la posibilidad de que se reduzca adicionalmente la demanda agregada (Greenwald y Stiglitz, 1987). Desde esta perspectiva, entonces, la política fiscal podría ser contra-cíclica.

Desde un punto de vista más práctico, se ha argumentado que la política fiscal es pro-cíclica debido a la disponibilidad de ingresos públicos a lo largo de las distintas fases del ciclo económico, incluyendo las facilidades en expansiones y las dificultades en recesiones para acceder al crédito, de modo que durante los auges el gasto público crecerá y bajará en las recesiones (Burnside y Meshcheryakova, 2005; Alesina et al., 2008; Blanchard et al., 2010). Más aun, en la lógica del Consenso de Washington y los programas de estabilización del Fondo Monetario Internacional adoptados en varios países en desarrollo, las crisis eran usualmente consecuencia de políticas expansivas que resultaban en excesos de demanda que llevaban a altas tasas de inflación y déficit externos y fiscales, entre otros desequilibrios. Para estabilizar la economía se recomendaban políticas restrictivas entre las que se contaban severos recortes del gasto público, entre otras medidas (Williamson, 2009). Recientemente se ha mantenido esta práctica con la idea de que se debe enviar señales a los mercados de que se llevarán a cabo los ajustes necesarios para preservar los equilibrios macroeconómicos (Blanchard et al., 2010; Galati y Moessner, 2013).

Aunque breve, esta revisión de literatura permite establecer que el gasto público puede ser pro o contra-cíclico, dependiendo de diferentes

Los salarios de eficiencia son superiores al de equilibrio bajo el supuesto de que fomentan la productividad de los trabajadores (Venegas y Rodríguez, 2009). 
situaciones. En el primer caso, no obstante, resulta evidente que el gasto público amplifica las fluctuaciones de la actividad productiva, especialmente en las recesiones cuando contribuye a profundizar la caída de la actividad productiva. En el segundo, por el contrario, un manejo contracíclico del gasto permite amortiguar esas fluctuaciones (aunque para ello se requiere de la acumulación de recursos en las fases de auge).

\section{Metodología eCONOMÉTRICA}

La metodología utilizada en este documento consta de dos etapas, sugeridas por Kydland y Prescott (1990), que se describen en las siguientes subsecciones. En la primera se explica cómo se identifica el indicador (estacionario) del ciclo, con base en una medida de actividad económica, mientras que en la segunda se expone la metodología para estimar el co-movimiento entre ese indicador y los componentes cíclicos del gasto público de los estados del centro de México, permitiendo la existencia de cambios estructurales en esa relación.

\subsection{Indicadores del ciclo económico}

En este documento se utilizan dos visiones del ciclo para identificar su indicador. En primer lugar, se parte de la visión de Lucas (1977: 3), quien define el ciclo como "los movimientos en torno a la tendencia del producto nacional bruto", y sostiene que el aspecto más relevante de su análisis se relaciona con "los movimientos comunes de las desviaciones, con respecto a una tendencia, de las distintas series temporales agregadas". En ese sentido, podría haber fluctuaciones por encima y por debajo de la tendencia subyacente del producto sin necesidad de que hubiese caídas en su nivel absoluto. Por ello, este enfoque se conoce como de ciclos de crecimiento. ${ }^{8}$

Entonces, con base en metodología empírica popularizada por Kydland y Prescott (1990), se utiliza el filtro introducido por Christiano y Fitzgerald (1999), denotado CF, para extraer el componente cíclico (estacionario) a partir de una serie de producción (y lo mismo se hace para las otras series de interés). Estos autores parten del supuesto de

8 Algunos estudios que ocupan esta metodología para analizar los ciclos son Mejía (2003), Cuadra (2008), Savva et al. (2010) y Loría y Salas (2014). 
que una serie de tiempo desestacionalizada $z_{t}$ está conformada por tres componentes estocásticos:

$$
z_{t}=\tau_{t}+c_{t}+\varepsilon_{t}
$$

donde $\tau_{t}$ es el componente tendencial, $c_{t}$ el componente cíclico estacionario y $\varepsilon_{t}$ el componente irregular también estacionario, para $t=1,2$, $\ldots, T$. Brevemente, el filtro CF, relaciona los distintos componentes de la serie de tiempo definidos en (1) con diferentes frecuencias, lo que le permite asignar los movimientos de baja frecuencia a la tendencia y los de alta frecuencia al término de perturbación, aislando los movimientos cíclicos en una banda con un rango específico de duración. ${ }^{9}$ Por lo tanto, permite obtener un componente cíclico más suave definido como:

$$
c_{t}=y_{t}-\tau_{t}-\varepsilon_{t}
$$

Además, el filtro CF es el más general dentro de los filtros de "paso de banda" que permite que cambien las ponderaciones de los adelantos y rezagos en su representación de medias móviles, por lo que se reduce el problema de inicio y final de la muestra que padecen otros (Valdivia y Yujra, 2009). Más aun, por estimar una tendencia suave y cambiante, permite captar, al menos parcialmente, los efectos de cambios estructurales que pueda presentar ésta y excluir las observaciones anómalas del componente cíclico al atribuirlo al término de ruido.

En segundo lugar, con base en la visión del ciclo clásico de Burns y Mitchell (1946), en la que el éste se identifica a partir de alzas y bajas en el nivel absoluto de las variables analizadas, se utilizan también sus tasas de crecimiento (TC), mismas que no solamente eliminan la tendencia subyacente de las series, sino que se pueden vincular a la sucesión de las fases de expansión y recesión del ciclo durante las cuales tomarán valores positivos y negativos, respectivamente, como sugieren Pérez et al. (2007). ${ }^{10}$ Cabe mencionar, además, que las tasas de crecimiento son una transformación comúnmente usada en el análisis económico.

9 Mejía et al. (2005) encuentran que el ciclo económico de México dura poco más de ocho años (100 meses en promedio), mientras que Erquizio (2007) sugiere que el ciclo mexicano dura entre 6 y 7 años (alrededor de 27 trimestres); ambas medidas caen en la duración asumida en la construcción de los filtros de eliminación de tendencia. 
Con estos dos métodos de eliminación de tendencia se tendrán resultados complementarios, aunque no idénticos: por un lado, los que corresponden a la frecuencia del ciclo (de crecimiento), y, por otro, los asociados a la convencional medida de crecimiento de las variables que se puede vincular al enfoque de los ciclos clásicos.

\subsection{Prueba de cambio estructural de Bai y Perron}

Para determinar si ha habido un cambio estructural en la relación del gasto público con el ciclo económico se utiliza la prueba de Bai y Perron (1998). Su metodología considera la posibilidad de que existan múltiples cambios estructurales en modelos de regresión lineal estimados secuencialmente a través de la minimización de la suma de los residuos al cuadrado. ${ }^{11}$ El modelo general sin cambios estructurales para dos variables estacionarias $x_{t}$ y $y_{t}$ puede definirse como:

$$
y_{t}=\beta_{0}+\beta_{1} x_{t}+\varepsilon_{t}
$$

donde $y_{t}$ es la variable dependiente observada, $x_{t}$ la variable explicatoria y $\varepsilon_{t}$ el término de perturbación que sigue un proceso ruido blanco; $\beta_{0}$ y $\beta_{1}$ denotan el intercepto y el coeficiente pendiente con valores constantes en la muestra. Se debe hacer notar que el tema de la causalidad no es relevante en este estudio, pues se trata de analizar solamente el co-movimiento entre las dos variables. En ese sentido, aunque se usa análisis de regresión, el coeficiente de correlación se puede obtener mediante una sencilla transformación. ${ }^{12}$

Por su parte, un modelo que considera $m$ cambios ( $m+1$ regímenes) se puede formular como:

10 En sentido estricto no se utiliza un enfoque clásico, lo cual requeriría precisamente de la identificación de las correspondientes fases de expansión y recesión. No obstante, el uso de tasas de crecimiento, además de aproximarlas a partir de sus valores positivos y negativos, tiene la ventaja de que permite la aplicación de métodos paramétricos para caracterizar alternativamente la relación entre las variables de interés.

11 El modelo de Bai y Perron (1998) puede ser usado para formas generales de autocorrelación y heteroscedasticidad en los errores, así como para variables dependientes rezagadas, regresores con tendencia y diferentes distribuciones de los errores.

12 La relación entre el coeficiente de correlación entre dos variables aleatorias $x$ y $y(r)$ y el coeficiente pendiente de la regresión entre las mismas $(b)$ puede expresarse como $r=b S_{x} / S_{y}$, donde $S_{x}$ y $S_{y}$ denotan las desviaciones estándar de $x$ y $y$, respectivamente. 


$$
y_{t}=\sum_{i=1}^{m+1} \alpha_{i} D_{i t}+\sum_{i=1}^{m+1} \beta_{i} D_{i t} x_{t}+\varepsilon_{t}
$$

para los regímenes $i=1, \ldots, m+1$, donde se usan los índices $\left(T_{1}, \ldots, T_{m}\right)$ para representar los puntos de quiebre desconocidos y, por convención, $T_{0}=0$ y $T_{m+1}=T$ para indicar el inicio y fin de la muestra; $D_{i t}$ es una variable dicótoma que toma los valores de 1 para el segmento de la muestra $T_{i-1}<t<T_{i}$ y cero para cualquier otro caso $(i=1, \ldots, m+1)$. El propósito es estimar los coeficientes de regresión desconocidos $\alpha_{i}$ y $\beta_{i}$ junto con los puntos de quiebre $T_{i}$ cuando $T$ observaciones de $x_{t} \mathrm{y} y_{t}$ están disponibles. Dado que el procedimiento es secuencial, se identifica al primer punto de cambio estructural como aquel en el que se minimiza la suma de residuos al cuadrado, denotado $\hat{T}_{l}$, así como los parámetros $\hat{\alpha_{1}}$ y $\beta_{1}$ y $\hat{\alpha_{2}}$ y $\beta_{2}$ del primero y segundo regímenes, tomando la muestra completa en la estimación. ${ }^{13}$ Como paso siguiente, la muestra se divide en dos segmentos $\left[1, \hat{T}_{1}\right]$ y $\left[\hat{T}_{1}, T_{m+1}\right]$ y en cada uno se realiza el mismo procedimiento para estimar un nuevo punto de cambio estructural, y así sucesivamente.

En general, como el método de estimación considerado se basa en el principio de mínimos cuadrados, para cada $m$-ésima partición $\left(T_{1}, \ldots, T_{m}\right)$ , denotado $\left\{T_{i}\right\}$, las estimaciones asociadas de $\alpha_{i}$ y $\beta_{i}$ se denotan como $\hat{\alpha}$ $\left(\left\{T_{i}\right\}\right)$ y $\beta\left(\left\{T_{i}\right\}\right)$. Sustituyendo éstas en la función objetivo y denotando el resultado de la suma de los residuos al cuadrado como $S_{T}\left(T_{1}, \ldots, T_{m}\right)$ y los puntos de quiebre estimados como $\left(\hat{T}_{1}, \ldots, \hat{T}_{m}\right)$, tenemos que

$$
\left(\hat{T}_{1}, \ldots, \widehat{T}_{m}\right)=\operatorname{argmin}_{T_{1}, \ldots, T_{m}} S_{T}\left(T_{1}, \ldots, T_{m}\right)
$$

donde la minimización es tomada sobre todas las particiones $\left(T_{1}, \ldots, T_{m}\right)$ tal que $T_{i}-T_{i-1} \geq q$. Así, los estimadores de los puntos de quiebre son los minimizadores globales de la función objetivo, en tanto que los parámetros estimados de la regresión están asociados a los mínimos cuadrados estimados de la m-ésima partición obtenida $\left\{T_{i}\right\}$, es decir $\hat{\alpha}_{i}=\hat{\alpha}\left(\left\{\hat{T}_{i}\right\}\right)$ y $\hat{\beta}_{i}=\beta\left(\left\{\hat{T}_{i}\right\}\right)$.

13 Convencionalmente se excluye el $15 \%$ de las observaciones al principio y al final de la muestra (trimming percentage) para tener los grados de libertad mínimos para estimar los parámetros del primero y último régimen, de modo que $\mathrm{T}_{1}$ y $\mathrm{T}_{\mathrm{m}}$ estarán en el intervalo $[0.15,0.85]$ de la muestra. 
Bai y Perron (1998) proponen una prueba estadística para verificar la hipótesis nula de existencia de $l$ quiebres versus la alternativa de $l+1$ quiebres, la cual permite determinar tanto el número como la fecha de los cambios estructurales, a diferencia de las otras dos pruebas generales que proponen; ${ }^{14}$ la prueba es de tipo razón de verosimilitud y sigue una distribución $F$. Para que se rechace la hipótesis nula, el valor mínimo total de la suma de los cuadrados de los residuos (sobre todos los segmentos en los que se incluye un quiebre adicional) debe ser suficientemente menor que la suma del cuadrado de los residuos del modelo con 1 quiebres, por lo que el estadístico de prueba se define como:

$$
\operatorname{supLR} R_{T}(l+1 \mid l)=\frac{s_{T}\left(\hat{T}_{1}, \ldots, \hat{T}_{l}\right)-s_{T}\left(\hat{1}_{1}, \ldots, \hat{T}_{l+1}\right)}{s_{T}\left(\hat{1}_{1}, \ldots, \hat{T}_{l+1}\right) T}
$$

En general, este estadístico mide la diferencia estadística entre la suma de los errores al cuadrado óptima del modelo con $l$ cambios estructurales y la óptima del modelo con $l+1$ cambios. Los valores críticos son calculados y reportados por Bai y Perron (2003).

\section{Resultados}

En esta sección se presentan los resultados sobre el grado de co-movimiento entre los componentes del gasto público y el indicador del ciclo, así como sobre los posibles cambios estructurales en dicha relación durante el periodo 1980-2015, para los estados del centro de México y para la economía en su conjunto. Las variables empleadas son el PIB, de donde se obtiene el indicador del ciclo, el gasto público total

14 Consideran también la prueba de no cambio estructural (hipótesis nula) versus algún número fijo de quiebres estructurales $\mathrm{m}=\mathrm{k}$ (hipótesis alternativa) y la prueba máxima doble, en la cual se prueba ningún cambio estructural versus un número desconocido de cambios, dado algún límite superior M (véase Bai y Perron, 1998). 
(GT) y sus componentes, entre los que se cuentan el gasto administrativo (GA), las transferencias (TR) y la obra pública (OP). ${ }^{15}$ En aras de la claridad, la nomenclatura para distinguir las distintas variables se compone de un prefijo para hacer referencia al estado (definido en el cuadro 1) seguido de la notación de la variable arriba citada y de un sufijo asociado al método de eliminación de tendencia que puede ser CF o TC. Por ejemplo, los componentes cíclicos obtenidos con el filtro CF de la obra pública del Estado de México y del gasto total nacional obtenido con la TC se denotarían MOPCF y NGTTC, respectivamente.

Algunas características básicas de las variables analizadas se muestran en el cuadro 1, el cual está dividido en varias secciones. En la primera se presenta la participación del PIB de cada estado cen el total nacional, donde se puede ver que el que más aporta es la Ciudad de México con $17.6 \%$ y el que menos es Tlaxcala con apenas $0.6 \%$. La suma total de la región es de alrededor de un tercio del PIB nacional, con lo que se corrobora su importancia. En la segunda parte se ven las proporciones de los componentes de gasto con respecto al PIB nacional y estatal; se aprecia que en promedio, el gasto total nacional alcanza el $6.1 \%$, cifra baja si se compara con las publicadas generalmente que rondan el $20 \%$ debido a que aquí se excluye el gasto ejercido directamente por el gobierno federal. ${ }^{16}$ En la región centro, el gasto alcanza un promedio de $7.6 \%$ del PIB. Entre sus componentes destacan las transferencias tanto por su participación en el PIB como en el total del gasto nacional y regional, lo que contrasta claramente con la baja participación de la obra pública. Además, cabe resaltar que la suma del gasto administrativo, las transferencias y la obra pública representa más del $80.0 \%$ del gasto total en todas las entidades.

15 Sólo se incluyen los rubros del gasto para los que se encontró información homogénea para el periodo muestral.

16 El gasto nacional se obtuvo aquí como la suma del gasto ejercido por los estados, incluyendo aportaciones y participaciones federales. 
空 윽 商市

흥하. 흠

กํ. 콜

을 을

웅 $\frac{0}{0} \stackrel{5}{3}$

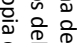

仓ิ

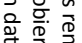

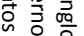

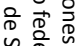



罗 $\frac{1}{2}$

을

증 总

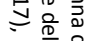

$\sum_{\mathrm{m}} \frac{\mathrm{D}}{\mathrm{D}} \frac{\mathrm{O}}{\mathrm{D}}$

응 믐

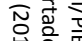

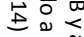

궃

늠

3ิ 3.

认่

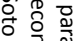

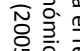

讪

市?

岗

กั้

ำ วิำ



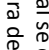

足

离

ขั

음.

ํㅡㄹ ํㅗำ

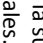

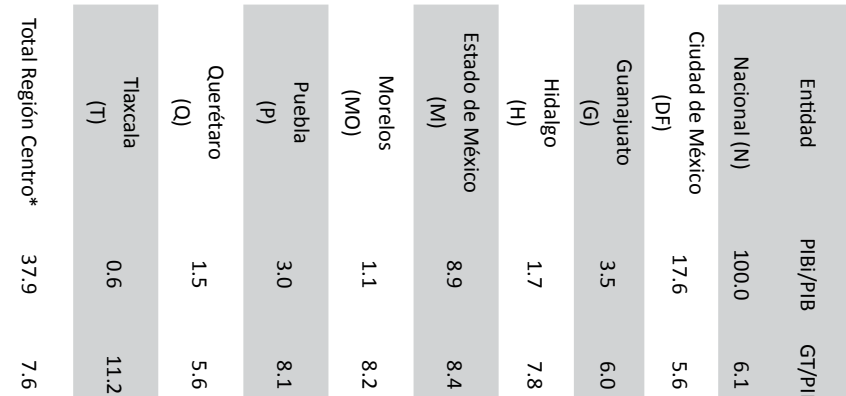

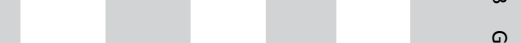

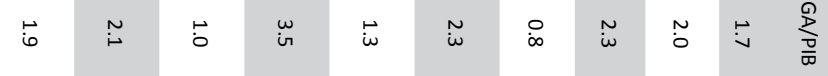

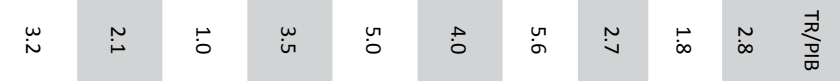

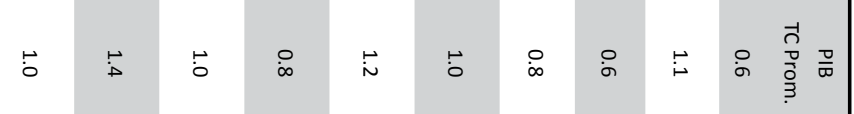

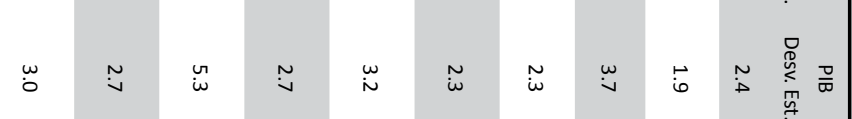

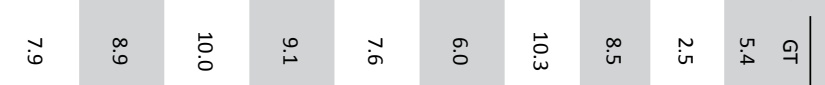

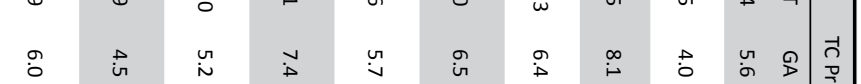

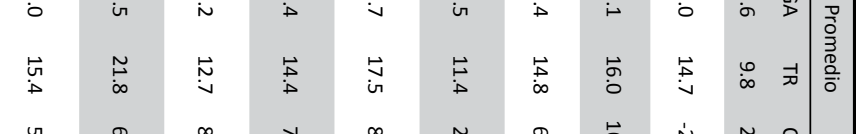

ज̆

$\stackrel{\vec{v}}{\vec{n}}$

$\stackrel{\bullet}{\dot{0}}$

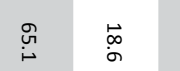

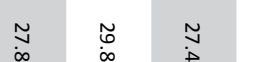

$\begin{array}{lll}\overrightarrow{8} & \overrightarrow{8} & \circ\end{array}$

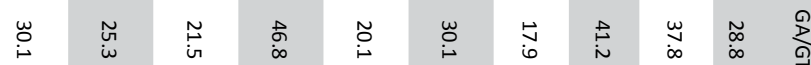

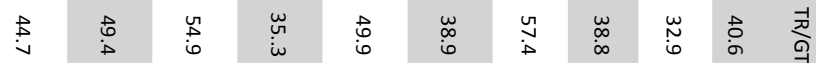

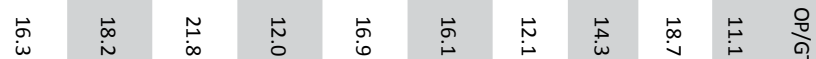

$\stackrel{\tilde{\omega}}{\sim}$

章

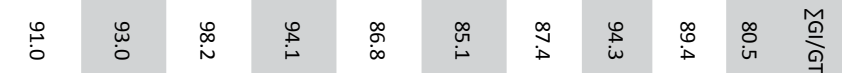


Por otro lado, en conjunto los estados de la región centro crecen al $3.0 \%$ anual en promedio, cifra superior a la tasa nacional en 0.6 puntos porcentuales. No obstante, el crecimiento de todos los estados presentan una volatilidad (medida por la desviación estándar) mayor que la del nacional, destacando Hidalgo y Guanajuato, con $7.4 \%$ y $7.2 \%$, respectivamente. A su vez, el rubro del gasto que más ha crecido en promedio en la región es el de transferencias con un $15.4 \%$ anual durante el periodo. Vale la pena subrayar que el crecimiento del gasto público presenta una volatilidad mucho mayor que la del PIB, lo que podría estar reflejando el alto grado de discrecionalidad en su manejo (Reyes y Mejía, 2012), lo que puede ser más severo a nivel estatal. En este rasgo, destacan las transferencias y la obra pública.

En la gráfica 1 se presenta el comportamiento de las tasas de crecimiento de las diferentes variables a nivel nacional donde se pueden destacar varios aspectos interesantes. El primero que llama la atención es el amplio rango de variación de las tasas de crecimiento de los diferentes rubros del gasto público ${ }^{17}$, así como la declinación de su volatilidad, especialmente desde los primeros años de la década de los años noventa, con excepción de la de la obra pública. Segundo, se aprecia una cierta correlación positiva entre el crecimiento del PIB y el de los diferentes indicadores del gasto hasta aproximadamente mediados de la misma década y una relación inversa en torno a la Gran Recesión, lo que se explica por la instrumentación de políticas contra-cíclicas por primera vez en décadas en la economía mexicana (Banxico, 2009; Villagómez y Navarro, 2010). Aunque con alzas y bajas más pronunciadas, los patrones de los componentes cíclicos de las variables obtenidos con el filtro CF, en general, son similares a las de las TC, como se muestra en la gráfica 2 .

17 Una posible explicación de la elevada volatilidad del gasto público es la alta discrecionalidad en su manejo, lo que se traduce en tasas de crecimiento erráticas y de alto valor, como Reyes y Mejía (2012) lo han sugerido. 
Gráfica 1

TASA DE CRECIMIENTO DEL PIB, GASTO TOTAL Y SUS COMPONENTES A NIVEL NACIONAL, 1980-2015

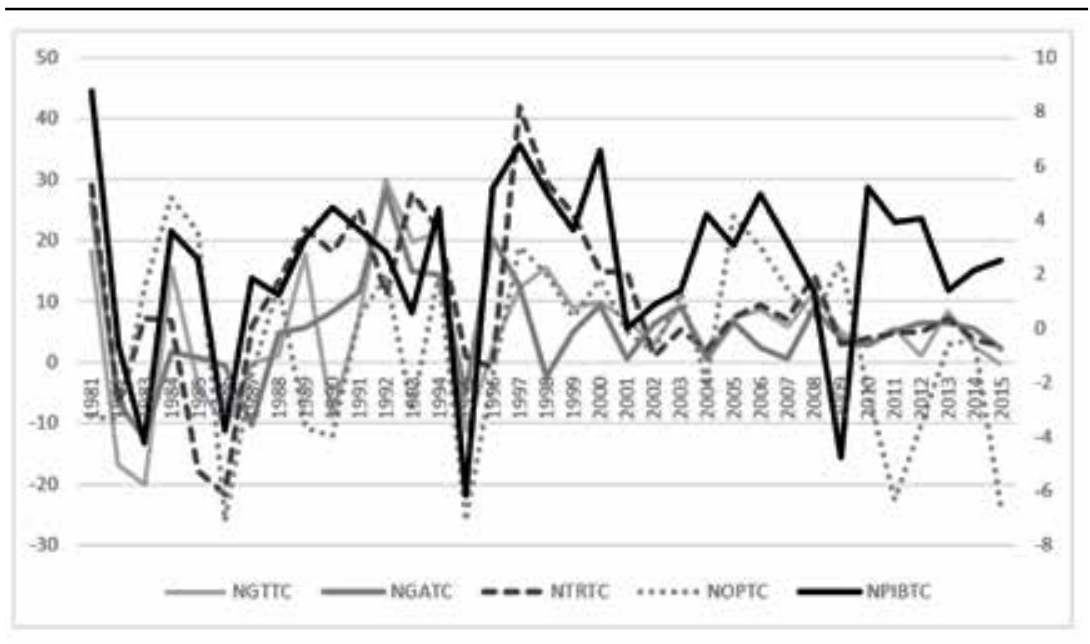

Fuente: Elaboración propia con datos de SIMBAD (2017), INEGI (2014) y German-Soto (2005).

Gráfica 2

COMPONENTES CÍ́CLICOS DEL FILTRO CF DEL PIB, GASTO TOTAL Y SUS DIFERENTES RUBROS A NIVEL NACIONAL, 1980-2015

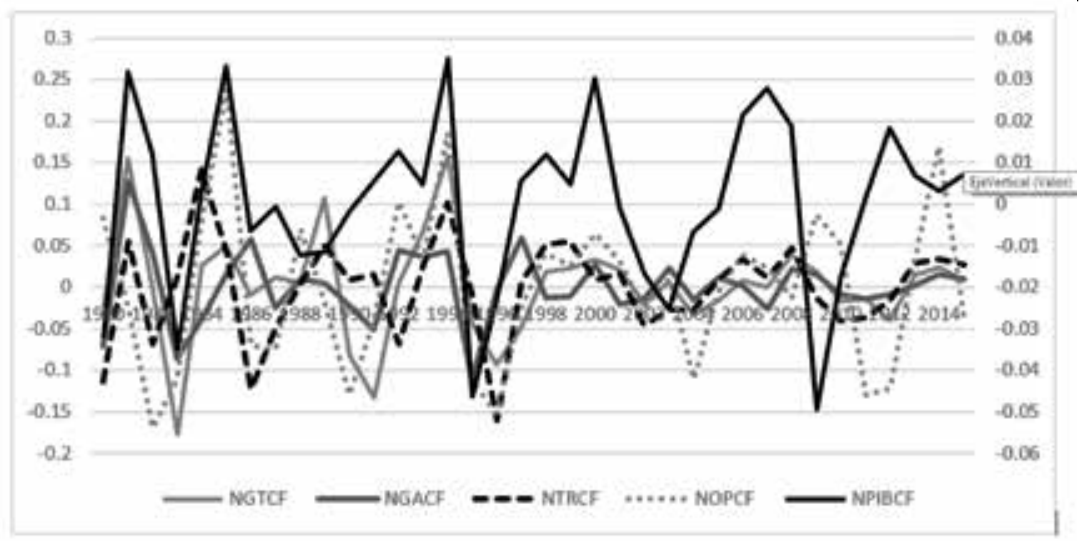

Fuente: Elaboración propia con datos de SIMBAD (2017), INEGI (2014) y German-Soto (2005).

Para estudiar la asociación entre las variables de interés, y sólo como referente inicial, se parte de la estimación de la relación lineal entre el indicador del ciclo económico del $j$-ésimo estado, $\mathrm{y}_{\mathrm{t}}^{\mathrm{j}}$, y el del $i$-ésimo 
componente del gasto del mismo estado, $\mathrm{g}_{\mathrm{it}}{ }^{\mathrm{j}}$, con base en la expresión (3), la cual se puede reformular como:

$$
y_{t}^{j}=\beta_{0}+\beta_{1} g_{i t}^{j}+\varepsilon_{t}
$$

Las estimaciones se muestran en el cuadro $2,{ }^{18}$ donde se puede ver, aunque con algunas excepciones, que los coeficientes-pendiente estimados por Mínimos Cuadrados Ordinarios (MCO) son positivos, pero no siempre significativos ni robustos al cambio de método de eliminación de tendencia. En el caso nacional, llama la atención la relación positiva y robusta, aunque con coeficientes de baja magnitud, de todos los componentes del gasto, con excepción de la obra pública que sólo es significativa al $10 \%$ en la frecuencia del ciclo económico. A este nivel, se podría aceptar la hipótesis de que la política fiscal nacional ha sido pro-cíclica y de importancia modesta, tal como se ha reportado en la literatura (Reyes y Mejía, 2012; Burnside y Meshcheryakova, 2005).

El análisis por estados, a su vez, sugiere relaciones positivas y robustas en la Ciudad de México, Hidalgo y el Estado de México, respecto a gasto total; la Ciudad de México, Hidalgo y Puebla en cuanto a gasto administrativo y, Morelos y Puebla referente a transferencias; en el resto de los estados, la gran mayoría de los coeficientes estimados son positivos, pero no significativos estadísticamente.

18 Las tasas de crecimiento son, en general, estacionarias de acuerdo a las pruebas de raíz unitaria de Kwiatkowski-Phillips-Schmidt-Shin (KPSS) y la de Mínimos Cuadrado Generalizados de Dickey Fuller (DF-GLS); estos resultados están disponibles a solicitud expresa. Por su parte, por construcción, los indicadores cíclicos obtenidos con el filtro CF son también estacionarios. 


\section{Cuadro 2}

ESTIMACIONES ECONOMÉTRICAS SIN CONSIDERAR CAMBIOS ESTRUCTURALES

\begin{tabular}{|c|c|c|c|c|c|c|c|}
\hline \multirow{2}{*}{$\begin{array}{c}\text { Var. } \\
\text { Dependiente }\end{array}$} & \multirow{2}{*}{ Entidad } & \multicolumn{3}{|c|}{ TC } & \multicolumn{3}{|c|}{ Filtro FC } \\
\hline & & C & Gasto Total & $\mathrm{R}^{2}$ & C & Gasto Total & $R^{2}$ \\
\hline \multirow{9}{*}{ PIB } & Nacional & $\begin{array}{c}1.507 \\
(0.004)\end{array}$ & $\begin{array}{c}0.162 \\
(0.006)\end{array}$ & 0.282 & $\begin{array}{c}0.000 \\
(0.987)\end{array}$ & $\begin{array}{c}0.157 \\
(0.004)\end{array}$ & 0.229 \\
\hline & $\begin{array}{l}\text { Ciudad de } \\
\text { México }\end{array}$ & $\begin{array}{c}1.32 \\
(0.050)\end{array}$ & $\begin{array}{c}0.236 \\
(0.000)\end{array}$ & 0.273 & $\begin{array}{c}0.001 \\
(0.882)\end{array}$ & $\begin{array}{c}0.185 \\
(0.009)\end{array}$ & 0.146 \\
\hline & Guanajuato & $\begin{array}{c}4.004 \\
(0.000)\end{array}$ & $\begin{array}{l}-0.034 \\
(0.728)\end{array}$ & 0.003 & $\begin{array}{l}0.001 \\
0.871)\end{array}$ & $\begin{array}{l}-0.107 \\
(0.164)\end{array}$ & 0.027 \\
\hline & Hidalgo & $\begin{array}{c}1.507 \\
(0.346)\end{array}$ & $\begin{array}{c}0.081 \\
(0.384)\end{array}$ & 0.025 & $\begin{array}{l}0.001 \\
(909)\end{array}$ & $\begin{array}{c}0.144 \\
(0.049)\end{array}$ & 0.071 \\
\hline & $\begin{array}{l}\text { Estado de } \\
\text { México }\end{array}$ & $\begin{array}{c}1.634 \\
(0.015)\end{array}$ & $\begin{array}{c}0.118 \\
(0.030)\end{array}$ & 0.112 & $\begin{array}{c}0.000 \\
(0.999)\end{array}$ & $\begin{array}{c}0.133 \\
(0.021)\end{array}$ & 0.097 \\
\hline & Morelos & $\begin{array}{c}2.486 \\
(0.011)\end{array}$ & $\begin{array}{c}0.097 \\
(0.351)\end{array}$ & 0.043 & $\begin{array}{c}0.000 \\
(0.932)\end{array}$ & $\begin{array}{c}0.175 \\
(0.058)\end{array}$ & 0.102 \\
\hline & Puebla & $\begin{array}{c}2.495 \\
(0.018)\end{array}$ & $\begin{array}{c}0.027 \\
(0.737)\end{array}$ & 0.004 & $\begin{array}{c}0.000 \\
(0.965)\end{array}$ & $\begin{array}{c}0.026 \\
(0.621)\end{array}$ & 0.003 \\
\hline & Querétaro & $\begin{array}{c}4.762 \\
(0.000)\end{array}$ & $\begin{array}{c}0.052 \\
(0.645)\end{array}$ & 0.020 & $\begin{array}{c}0.000 \\
(0.923)\end{array}$ & $\begin{array}{c}0.064 \\
(0.515)\end{array}$ & 0.024 \\
\hline & Tlaxcala & $\begin{array}{c}1.779 \\
(0.055)\end{array}$ & $\begin{array}{c}0.107 \\
(0.093)\end{array}$ & 0.121 & $\begin{array}{l}-0.001 \\
(0.895)\end{array}$ & $\begin{array}{c}0.066 \\
(0.352)\end{array}$ & 0.036 \\
\hline $\begin{array}{c}\text { Var. } \\
\text { Dependiente }\end{array}$ & Entidad & C & $\begin{array}{c}\text { Gasto } \\
\text { Administrativo }\end{array}$ & $\mathrm{R}^{2}$ & C & $\begin{array}{c}\text { Gasto } \\
\text { Administrativo }\end{array}$ & $\mathrm{R}^{2}$ \\
\hline \multirow{9}{*}{ PIB } & Nacional & $\begin{array}{c}1.293 \\
(0.036)\end{array}$ & $\begin{array}{c}0.196 \\
(0.007)\end{array}$ & 0.269 & $\begin{array}{c}0.000 \\
(0.989)\end{array}$ & $\begin{array}{c}0.257 \\
(0.000)\end{array}$ & 0.27 \\
\hline & $\begin{array}{l}\text { Ciudad de } \\
\text { México }\end{array}$ & $\begin{array}{c}1.560 \\
(0.069)\end{array}$ & $\begin{array}{c}0.088 \\
(0.201)\end{array}$ & 0.074 & $\begin{array}{c}0.001 \\
(0.875)\end{array}$ & $\begin{array}{c}0.138 \\
(0.002)\end{array}$ & 0.207 \\
\hline & Guanajuato & $\begin{array}{c}4.034 \\
(0.005)\end{array}$ & $\begin{array}{l}-0.039 \\
(0.352)\end{array}$ & 0.010 & $\begin{array}{c}0.001 \\
(0.869)\end{array}$ & $\begin{array}{l}-0.047 \\
(0.357)\end{array}$ & 0.010 \\
\hline & Hidalgo & $\begin{array}{c}1.135 \\
(0.366)\end{array}$ & $\begin{array}{c}0.188 \\
(0.053)\end{array}$ & 0.142 & $\begin{array}{c}0.001 \\
(0.916)\end{array}$ & $\begin{array}{c}0.176 \\
(0.048)\end{array}$ & 0.134 \\
\hline & $\begin{array}{l}\text { Estado de } \\
\text { México }\end{array}$ & $\begin{array}{c}1.592 \\
(0.080)\end{array}$ & $\begin{array}{c}0.116 \\
(0.114)\end{array}$ & 0.090 & $\begin{array}{c}0.000 \\
(0.962)\end{array}$ & $\begin{array}{c}0.080 \\
(0.344)\end{array}$ & 0.036 \\
\hline & Morelos & $\begin{array}{c}2.877 \\
(0.009)\end{array}$ & $\begin{array}{c}0.060 \\
(0.330)\end{array}$ & 0.029 & $\begin{array}{c}0.000 \\
(0.983)\end{array}$ & $\begin{array}{c}0.108 \\
(0.126)\end{array}$ & 0.073 \\
\hline & Puebla & $\begin{array}{c}1.948 \\
(0.010)\end{array}$ & $\begin{array}{c}0.107 \\
(0.001)\end{array}$ & 0.205 & $\begin{array}{c}0.000 \\
(0.973)\end{array}$ & $\begin{array}{c}0.125 \\
(0.012)\end{array}$ & 0.134 \\
\hline & Querétaro & $\begin{array}{c}5.387 \\
(0.000)\end{array}$ & $\begin{array}{l}-0.021 \\
(0.758)\end{array}$ & 0.002 & $\begin{array}{c}0.000 \\
(0.939)\end{array}$ & $\begin{array}{l}-0.015 \\
(0.823)\end{array}$ & 0.001 \\
\hline & Tlaxcala & $\begin{array}{c}2.596 \\
(0.002)\end{array}$ & $\begin{array}{c}0.031 \\
(0.750)\end{array}$ & 0.004 & $\begin{array}{l}-0.001 \\
(0.890)\end{array}$ & $\begin{array}{c}0.073 \\
(0.392)\end{array}$ & 0.026 \\
\hline
\end{tabular}

Valores significativos al $5 \%$ en negritas y significativos al $10 \%$ en cursivas. Valores p entre paréntesis. Fuente: estimaciones propias. 


\section{Cuadro 2 (Continuación)}

\section{ESTIMACIONES ECONOMÉTRICAS SIN CONSIDERAR CAMBIOS ESTRUCTURALES}

\begin{tabular}{|c|c|c|c|c|c|c|c|}
\hline $\begin{array}{c}\text { Var. } \\
\text { Dependiente }\end{array}$ & Entidad & C & Transferencias & $\mathrm{R}^{2}$ & $\mathrm{C}$ & Transferencias & $\mathrm{R}^{2}$ \\
\hline \multirow{9}{*}{ PIB } & Nacional & $\begin{array}{c}1.178 \\
(0.055)\end{array}$ & $\begin{array}{c}0.122 \\
(0.000)\end{array}$ & 0.235 & $\begin{array}{c}0.000 \\
(0.925)\end{array}$ & $\begin{array}{c}0.157 \\
(0.001)\end{array}$ & 0.192 \\
\hline & $\begin{array}{l}\text { Ciudad de } \\
\text { México }\end{array}$ & $\begin{array}{c}2.275 \\
(0.009)\end{array}$ & $\begin{array}{l}-0.025 \\
(0.579)\end{array}$ & 0.025 & $\begin{array}{c}0.001 \\
(0.882)\end{array}$ & $\begin{array}{c}0.007 \\
(0.830)\end{array}$ & 0.001 \\
\hline & Guanajuato & $\begin{array}{c}3.572 \\
(0.006)\end{array}$ & $\begin{array}{c}0.009 \\
(0.355)\end{array}$ & 0.004 & $\begin{array}{c}0.001 \\
(0.873)\end{array}$ & $\begin{array}{l}-0.008 \\
(0.673)\end{array}$ & 0.001 \\
\hline & Hidalgo & $\begin{array}{c}1.792 \\
(0.133)\end{array}$ & $\begin{array}{c}0.037 \\
(0.424)\end{array}$ & 0.021 & $\begin{array}{c}0.001 \\
(0.918)\end{array}$ & $\begin{array}{c}0.102 \\
(0.215)\end{array}$ & 0.067 \\
\hline & $\begin{array}{l}\text { Estado de } \\
\text { México }\end{array}$ & $\begin{array}{c}2.027 \\
(0.029)\end{array}$ & $\begin{array}{c}0.028 \\
(0.481)\end{array}$ & 0.021 & $\begin{array}{c}0.000 \\
(0.963)\end{array}$ & $\begin{array}{c}0.024 \\
(0.613)\end{array}$ & 0.009 \\
\hline & Morelos & $\begin{array}{c}2.805 \\
(0.011)\end{array}$ & $\begin{array}{c}0.024 \\
(0.208)\end{array}$ & 0.026 & $\begin{array}{c}0.000 \\
(0.962)\end{array}$ & $\begin{array}{c}0.094 \\
(0.014)\end{array}$ & 0.153 \\
\hline & Puebla & $\begin{array}{c}2.34 \\
(0.006)\end{array}$ & $\begin{array}{c}0.036 \\
(0.068)\end{array}$ & 0.046 & $\begin{array}{c}0.000 \\
(0.939)\end{array}$ & $\begin{array}{c}0.072 \\
(0.008)\end{array}$ & 0.102 \\
\hline & Querétaro & $\begin{array}{c}4.574 \\
(0.000)\end{array}$ & $\begin{array}{c}0.011 \\
(0.693)\end{array}$ & 0.002 & $\begin{array}{c}0.001 \\
(0.836)\end{array}$ & $\begin{array}{l}-0.043 \\
(0.506)\end{array}$ & 0.011 \\
\hline & Tlaxcala & $\begin{array}{c}2.594 \\
(0.004)\end{array}$ & $\begin{array}{c}0.006 \\
(0.463)\end{array}$ & 0.008 & $\begin{array}{l}-0.001 \\
(0.894)\end{array}$ & $\begin{array}{l}-0.004 \\
(0.834)\end{array}$ & 0.001 \\
\hline $\begin{array}{c}\text { Var. } \\
\text { Dependiente }\end{array}$ & Entidad & C & Obras Públicas & $\mathrm{R}^{2}$ & C & Obras Públicas & $\mathrm{R}^{2}$ \\
\hline \multirow{9}{*}{ PIB } & Nacional & $\begin{array}{c}2.245 \\
(0.001)\end{array}$ & $\begin{array}{c}0.049 \\
(-0.288)\end{array}$ & 0.048 & $\begin{array}{c}0.000 \\
(0.937)\end{array}$ & $\begin{array}{c}0.063 \\
(0.096)\end{array}$ & 0.082 \\
\hline & $\begin{array}{l}\text { Ciudad de } \\
\text { México }\end{array}$ & $\begin{array}{c}1.929 \\
(0.015)\end{array}$ & $\begin{array}{c}0.007 \\
(0.795)\end{array}$ & 0.001 & $\begin{array}{c}0.001 \\
(0.876)\end{array}$ & $\begin{array}{l}-0.023 \\
(0.462)\end{array}$ & 0.012 \\
\hline & Guanajuato & $\begin{array}{c}3.919 \\
(0.019)\end{array}$ & $\begin{array}{l}-0.002 \\
(0.365)\end{array}$ & 0.008 & $\begin{array}{c}0.001 \\
(0.873)\end{array}$ & $\begin{array}{c}0.001 \\
(0.963)\end{array}$ & 0.000 \\
\hline & Hidalgo & $\begin{array}{c}2.439 \\
(0.030)\end{array}$ & $\begin{array}{l}-0.015 \\
(0.718)\end{array}$ & 0.002 & $\begin{array}{c}0.001 \\
(0.908)\end{array}$ & $\begin{array}{c}0.021 \\
(0.575)\end{array}$ & 0.004 \\
\hline & $\begin{array}{l}\text { Estado de } \\
\text { México }\end{array}$ & $\begin{array}{c}2.268 \\
(0.005)\end{array}$ & $\begin{array}{c}0.038 \\
(0.389)\end{array}$ & 0.028 & $\begin{array}{c}0.000 \\
(0.950)\end{array}$ & $\begin{array}{l}-0.034 \\
(0.240)\end{array}$ & 0.035 \\
\hline & Morelos & $\begin{array}{c}2.919 \\
(0.037)\end{array}$ & $\begin{array}{c}0.002 \\
(0.483)\end{array}$ & 0.028 & $\begin{array}{c}0.000 \\
(0.960)\end{array}$ & $\begin{array}{c}0.010 \\
(0.732)\end{array}$ & 0.002 \\
\hline & Puebla & $\begin{array}{c}2.358 \\
(0.012)\end{array}$ & $\begin{array}{c}0.011 \\
(0.605)\end{array}$ & 0.005 & $\begin{array}{c}0.002 \\
(0.774)\end{array}$ & $\begin{array}{l}-0.012 \\
(0.595)\end{array}$ & 0.004 \\
\hline & Querétaro & $\begin{array}{c}5.416 \\
(0.000)\end{array}$ & $\begin{array}{l}-0.016 \\
(0.540)\end{array}$ & 0.007 & $\begin{array}{c}0.000 \\
(0.953)\end{array}$ & $\begin{array}{l}-0.031 \\
(0.229)\end{array}$ & 0.033 \\
\hline & Tlaxcala & $\begin{array}{c}2.828 \\
(0.006)\end{array}$ & $\begin{array}{c}-0.014 \\
(0.613)\end{array}$ & 0.007 & $\begin{array}{c}-0.001 \\
(0.891)\end{array}$ & $\begin{array}{c}0.014 \\
(0.516)\end{array}$ & 0.011 \\
\hline
\end{tabular}

Valores significativos al $5 \%$ en negritas y significativos al $10 \%$ en cursivas. Valores $p$ entre paréntesis.

Fuente: estimaciones propias. 
Sin embargo, como se ha mencionado previamente, es posible que las relaciones entre estas variables hayan cambiado en el transcurso del tiempo como consecuencia de los ajustes de las políticas de estabilización o de los efectos de las diferentes reformas estructurales que se han llevado a cabo en la economía mexicana durante las últimas décadas. Para analizar esta posibilidad se aplica la prueba de $l$ versus $l+l$ quiebres estructurales sugerida por Bai y Perron (1998) en el marco del modelo definido en la expresión (4). Las hipótesis evaluadas, los valores de los estadísticos de prueba y las fechas sugeridas de los quiebres se presentan en el cuadro 3 .

\section{Cuadro 3}

PRUEBA $L$ VS. $L+1$ QUIEBRES DE BAI Y PERRON ${ }^{\dagger}$

\begin{tabular}{|c|c|c|c|c|c|c|c|}
\hline \multicolumn{8}{|c|}{ Gasto Total } \\
\hline \multirow{2}{*}{ Entidad } & \multirow{2}{*}{$\begin{array}{l}\text { Prueba de } \\
\text { quiebres }\end{array}$} & \multicolumn{2}{|c|}{ TC } & \multirow{2}{*}{ Entidad } & \multirow{2}{*}{$\begin{array}{l}\text { Prueba de } \\
\text { quiebres }\end{array}$} & \multicolumn{2}{|c|}{$\mathrm{CF}$} \\
\hline & & $\mathrm{F}$ & Fechas & & & $\mathrm{F}$ & Fechas \\
\hline \multirow{4}{*}{ Nacional } & 0 vs. 1 & 2.01 & - & \multirow{4}{*}{ Nacional } & 0 vs. 1 & $11.53^{*}$ & 2009 \\
\hline & 1 vs. 2 & - & - & & 1 vs. 2 & $22.35^{*}$ & 2004 \\
\hline & 2 vs. 3 & - & - & & 2 vs. 3 & $69.89 *$ & 1998 \\
\hline & 3 vs. 4 & - & - & & 3 vs. 4 & 0.90 & - \\
\hline $\begin{array}{l}\text { Ciudad de } \\
\text { México }\end{array}$ & 0 vs. 1 & 2.36 & - & $\begin{array}{l}\text { Ciudad de } \\
\text { México }\end{array}$ & 0 vs. 1 & 2.58 & - \\
\hline Guanajuato & 0 vs. 1 & 1.45 & - & Guanajuato & 0 vs. 1 & 0.38 & - \\
\hline Hidalgo & 0 vs. 1 & 1.21 & - & Hidalgo & 0 vs. 1 & 0.81 & - \\
\hline \multirow{2}{*}{$\begin{array}{l}\text { Estado de } \\
\text { México }\end{array}$} & 0 vs. 1 & $8.13^{*}$ & 1986 & \multirow{2}{*}{$\begin{array}{l}\text { Estado de } \\
\text { México }\end{array}$} & 0 vs. 1 & 3.12 & - \\
\hline & 1 vs. 2 & 1.62 & - & & 1 vs. 2 & - & - \\
\hline \multirow{2}{*}{ Morelos } & 0 vs. 1 & 0.56 & - & \multirow{2}{*}{ Morelos } & 0 vs. 1 & $9.10 *$ & 2011 \\
\hline & 1 vs. 2 & - & - & & 1 vs. 2 & 0.36 & - \\
\hline Puebla & 0 vs. 1 & 1.32 & - & Puebla & 0 vs. 1 & 2.32 & - \\
\hline \multirow{3}{*}{ Querétaro } & 0 vs. 1 & $6.36^{*}$ & 1987 & \multirow{3}{*}{ Querétaro } & 0 vs. 1 & $29.93 *$ & 1987 \\
\hline & 1 vs. 2 & 1.06 & - & & 1 vs. 2 & $12.53^{*}$ & 1993 \\
\hline & 2 vs. 3 & - & - & & 2 vs. 3 & 1.28 & - \\
\hline \multirow{2}{*}{ Tlaxcala } & 0 vs. 1 & $7.99 *$ & 1986 & \multirow{2}{*}{ Tlaxcala } & 0 vs. 1 & 4.50 & - \\
\hline & 1 vs. 2 & 4.51 & - & & 1 vs. 2 & - & - \\
\hline \multicolumn{8}{|c|}{ Gasto Administrativo } \\
\hline \multirow{3}{*}{ Nacional } & 0 vs. 1 & 3.68 & - & \multirow{3}{*}{ Nacional } & 0 vs. 1 & $22.43^{*}$ & 2003 \\
\hline & 1 vs. 2 & - & - & & 1 vs. 2 & $39.27 *$ & 1998 \\
\hline & 2 vs. 3 & - & - & & 2 vs. 3 & 3.24 & - \\
\hline
\end{tabular}




\section{Cuadro 3 (Continuación) \\ Prueba $L$ Vs. $l+1$ Quiebres de Bal y Perron ${ }^{\dagger}$}

\begin{tabular}{|c|c|c|c|c|c|c|c|}
\hline Ciudad de & 0 vs. 1 & $6.70^{*}$ & 1987 & \multirow{2}{*}{$\begin{array}{l}\text { Ciudad de } \\
\text { México }\end{array}$} & 0 vs. 1 & 2.78 & - \\
\hline México & 1 vs. 2 & 2.80 & & & 1 vs. 2 & - & - \\
\hline \multirow{2}{*}{ Guanajuato } & 0 vs. 1 & 2.90 & - & \multirow{2}{*}{ Guanajuato } & 0 vs. 1 & $6.22^{*}$ & 1986 \\
\hline & 1 vs. 2 & - & - & & 1 vs. 2 & 5.26 & - \\
\hline Hidalgo & 0 vs. 1 & 1.48 & - & Hidalgo & 0 vs. 1 & 1.16 & - \\
\hline \multirow{2}{*}{$\begin{array}{l}\text { Estado de } \\
\text { México }\end{array}$} & 0 vs. 1 & 4.58 & - & \multirow{2}{*}{$\begin{array}{l}\text { Estado de } \\
\text { México }\end{array}$} & 0 vs. 1 & $7.35^{*}$ & 1998 \\
\hline & 1 vs. 2 & - & - & & 1 vs. 2 & 2.78 & - \\
\hline \multirow{2}{*}{ Morelos } & 0 vs. 1 & 2.68 & - & \multirow{2}{*}{ Morelos } & 0 vs. 1 & $21.51^{*}$ & 1986 \\
\hline & 1 vs. 2 & - & - & & 1 vs. 2 & 1.47 & - \\
\hline \multirow{2}{*}{ Puebla } & 0 vs. 1 & $35.91 *$ & 1987 & \multirow{2}{*}{ Puebla } & 0 vs. 1 & $30.13^{*}$ & 1986 \\
\hline & 1 vs. 2 & 1.94 & - & & 1 vs. 2 & 4.50 & - \\
\hline Querétaro & 0 vs. 1 & 0.92 & - & Querétaro & 0 vs. 1 & 0.63 & - \\
\hline \multirow{2}{*}{ Tlaxcala } & 0 vs. 1 & $9.38^{*}$ & 1986 & \multirow{2}{*}{ Tlaxcala } & 0 vs. 1 & 5.43 & - \\
\hline & 1 vs. 2 & 3.88 & - & & 1 vs. 2 & - & - \\
\hline \multicolumn{8}{|c|}{ Transferencias } \\
\hline Nacional & 0 vs. 1 & 1.10 & - & Nacional & 0 vs. 1 & 1.67 & - \\
\hline \multirow{4}{*}{$\begin{array}{l}\text { Ciudad de } \\
\text { México }\end{array}$} & 0 vs. 1 & $14.80^{*}$ & 1986 & \multirow{4}{*}{$\begin{array}{l}\text { Ciudad de } \\
\text { México }\end{array}$} & 0 vs. 1 & $8.05^{*}$ & 1985 \\
\hline & 1 vs. 2 & $10.00^{*}$ & 1996 & & 1 vs. 2 & 1.17 & - \\
\hline & 2 vs. 3 & $11.35^{*}$ & 2001 & & 2 vs. 3 & - & - \\
\hline & 3 vs. 4 & 1.94 & - & & 3 vs. 4 & - & - \\
\hline Guanajuato & 0 vs. 1 & 0.61 & - & Guanajuato & 0 vs. 1 & 1.11 & - \\
\hline \multirow{2}{*}{ Hidalgo } & 0 vs. 1 & $9.15^{*}$ & 1987 & \multirow{2}{*}{ Hidalgo } & 0 vs. 1 & $22.19^{*}$ & 1986 \\
\hline & 1 vs. 2 & 1.99 & - & & 1 vs. 2 & 5.44 & - \\
\hline \multirow{2}{*}{$\begin{array}{l}\text { Estado de } \\
\text { México }\end{array}$} & 0 vs. 1 & 1.69 & - & \multirow{2}{*}{$\begin{array}{l}\text { Estado de } \\
\text { México }\end{array}$} & 0 vs. 1 & $7.44^{*}$ & 2000 \\
\hline & 1 vs. 2 & - & - & & 1 vs. 2 & 4.10 & - \\
\hline \multirow{2}{*}{ Morelos } & 0 vs. 1 & $6.17^{*}$ & 1986 & \multirow{2}{*}{ Morelos } & 0 vs. 1 & $45.34^{*}$ & 1985 \\
\hline & 1 vs. 2 & 5.88 & - & & 1 vs. 2 & 3.37 & - \\
\hline \multirow{2}{*}{ Puebla } & 0 vs. 1 & 1.88 & - & \multirow{2}{*}{ Puebla } & 0 vs. 1 & $7.01 *$ & 2012 \\
\hline & 1 vs. 2 & - & - & & 1 vs. 2 & 4.22 & - \\
\hline \multirow{2}{*}{ Querétaro } & 0 vs. 1 & $9.90 *$ & 1987 & \multirow{2}{*}{ Querétaro } & 0 vs. 1 & $14.16^{*}$ & 1987 \\
\hline & 1 vs. 2 & 0.65 & - & & 1 vs. 2 & 2.31 & - \\
\hline \multirow{5}{*}{ Tlaxcala } & 0 vs. 1 & $48.81^{*}$ & 1987 & \multirow{5}{*}{ Tlaxcala } & 0 vs. 1 & $36.03^{*}$ & 1986 \\
\hline & 1 vs. 2 & $11.49 *$ & 1996 & & 1 vs. 2 & $27.41^{*}$ & 2002 \\
\hline & 2 vs. 3 & $7.45^{*}$ & 2001 & & 2 vs. 3 & $22.11^{*}$ & 1992 \\
\hline & 3 vs. 4 & 5.14 & - & & 3 vs. 4 & $28.58^{*}$ & 1997 \\
\hline & 4 vs. 5 & - & - & & 4 vs. 5 & 6.58 & - \\
\hline
\end{tabular}


Cuadro 3 (Continuación)

Prueba L Vs. L+1 Quiebres de Bal y Perron ${ }^{\dagger}$

\begin{tabular}{|c|c|c|c|c|c|c|c|}
\hline \multicolumn{8}{|c|}{ Obra Pública } \\
\hline \multirow{2}{*}{ Nacional } & 0 vs. 1 & 4.23 & - & \multirow{2}{*}{ Nacional } & 0 vs. 1 & $6.10^{*}$ & 2009 \\
\hline & 1 vs. 2 & - & - & & 1 vs. 2 & 3.66 & - \\
\hline \multirow{3}{*}{$\begin{array}{l}\text { Ciudad de } \\
\text { México }\end{array}$} & 0 vs. 1 & $6.35^{*}$ & 1987 & \multirow{3}{*}{$\begin{array}{l}\text { Ciudad de } \\
\text { México }\end{array}$} & 0 vs. 1 & 14.57 & 1985 \\
\hline & 1 vs. 2 & $6.56^{*}$ & 1993 & & 1 vs. 2 & 3.15 & - \\
\hline & 2 vs. 3 & 3.60 & - & & 2 vs. 3 & - & - \\
\hline Guanajuato & 0 vs. 1 & 5.41 & - & Guanajuato & 0 vs. 1 & 1.33 & - \\
\hline \multirow{2}{*}{ Hidalgo } & 0 vs. 1 & $19.88^{*}$ & 1988 & \multirow{2}{*}{ Hidalgo } & 0 vs. 1 & 0.64 & - \\
\hline & 1 vs. 2 & 1.95 & & & 1 vs. 2 & - & - \\
\hline \multirow{4}{*}{$\begin{array}{l}\text { Estado de } \\
\text { México }\end{array}$} & 0 vs. 1 & $5.93 *$ & 1986 & \multirow{4}{*}{$\begin{array}{l}\text { Estado de } \\
\text { México }\end{array}$} & 0 vs. 1 & 4.27 & - \\
\hline & 1 vs. 2 & $17.63^{*}$ & 1996 & & 1 vs. 2 & - & - \\
\hline & 2 vs. 3 & $12.49 *$ & 2001 & & 2 vs. 3 & - & - \\
\hline & 3 vs. 4 & 5.30 & & & 3 vs. 4 & - & - \\
\hline \multirow{3}{*}{ Morelos } & 0 vs. 1 & $5.84 *$ & 1987 & \multirow{3}{*}{ Morelos } & 0 vs. 1 & $136.60^{*}$ & 1986 \\
\hline & 1 vs. 2 & $17.00^{*}$ & 1993 & & 1 vs. 2 & 2.11 & - \\
\hline & 2 vs. 3 & $0.60 *$ & & & 2 vs. 3 & - & - \\
\hline Puebla & 0 vs. 1 & 2.34 & - & Puebla & 0 vs. 1 & 2.93 & - \\
\hline \multirow{2}{*}{ Querétaro } & 0 vs. 1 & 1.13 & - & \multirow{2}{*}{ Querétaro } & 0 vs. 1 & $7.17^{*}$ & 2011 \\
\hline & 1 vs. 2 & - & - & & 1 vs. 2 & 1.06 & - \\
\hline \multirow{2}{*}{ Tlaxcala } & 0 vs. 1 & $9.99 *$ & 1986 & \multirow{2}{*}{ Tlaxcala } & 0 vs. 1 & 2.18 & - \\
\hline & 1 vs. 2 & 4.84 & - & & 1 vs. 2 & - & - \\
\hline
\end{tabular}

† Los valores críticos (al $5 \%$ de significancia) de los quiebres secuenciales de Bai y Perron (2003) son de la siguiente manera: para 0 vs. 1 es 11.47, 1 vs. 2 es 12.95, 2 vs. 3 es 14.03, 3 vs. 4 es 14.85 y 4 vs. 5 es 15.29, de igual forma aplica para todos los casos. * Significativo al $5 \%$.

Fuente: estimaciones propias.

En general, se observa la presencia de múltiples quiebres estructurales, los cuales no se presentan necesariamente en las mismas fechas cuando cambia el método de eliminación de tendencia. De hecho, se detectan más quiebres cuando se usa el filtro $\mathrm{CF}$ que cuando se trabaja con las TC. Específicamente, a nivel nacional, no se identifican quiebres cuando se usan las tasas de crecimiento de las variables. En cambio, ocurren varios en la frecuencia del ciclo, cuando se usan datos derivados del filtro CF: una proporción importante de ellos ocurre durante la segunda mitad de los años noventa, cuando se transita claramente de una economía altamente inestable, con elevadas tasas de inflación y frecuentes devaluaciones cambiarias, fugas de capitales, alta volatilidad 
del precio del petróleo y bajo o nulo crecimiento, a una economía más estable. En ese proceso, además, se pasa de la aplicación de programas de estabilización basados, entre otras medidas, en elevados recortes del gasto público, al menos hasta la crisis de 1995, a un manejo prudencial del mismo fundamentado esencialmente en la disponibilidad de recursos fiscales. ${ }^{19}$ Por otro lado, algunos quiebres ocurren en torno a la recesión de 2001-2003, mientras que otros más se presentan alrededor de la Gran Recesión de 2008-2009. Interesantemente en los modelos del gasto total, administrativo y de obra púbica nacionales se presentan quiebres en 2008 o 2009, lo que bien podría corresponder al cambio de orientación de la política fiscal para combatir la crisis, como sostienen Villagómez y Navarro (2010).

A nivel de estados, por otro lado, las fechas de los quiebres corresponden a periodos diferentes al caso nacional, ya que la mayoría se encuentran en la década de los años ochenta (principalmente entre 1985 y 1987), aunque algunos otros ocurren en los años noventa y sólo unos cuantos en torno a la Gran Recesión o a los años inmediatamente posteriores. Resulta importante menciones que las fechas de estos quiebres podrían estar asociados a cambios en la Ley de Coordinación Fiscal (1998 y 2007) y a los efectos de la Gran Recesión en los fondos participables a los estados. En el primer caso, se crearon los fondos de aportaciones federales (Ramo 33) en el marco de la descentralización del Estado; la educación y salud pasaron formalmente a ser funciones de las entidades federativas, por lo que los rubros más importantes del Ramo 33 van destinados a estos conceptos. En el segundo caso, la Recaudación Federal Participable (RFP), de donde se toman los recursos para participaciones y aportaciones, disminuyó por la recesión, por lo que se activó el Fondo de Estabilización de los Ingresos Fiscales), llevando a una mayor asignación de recursos a las entidades federativas y municipios para amortiguar la caída en la RFP. ${ }^{20}$

Entonces, un primer resultado relevante es que la relación gasto público y ciclo económico no ha sido estable en el periodo analizado, ni en la frecuencia de los ciclos de crecimiento ni en los ciclos clásicos. Eso indica que la relación lineal entre las variables analizadas cambia

\footnotetext{
Véanse Esquivel (2010) y Burnside y Meshcheryakova (2005) para análisis de estos procesos.

20 En 2013 se hizo una nueva reforma, pero la porción de la muestra que se deja en los extremos del periodo analizado no permite evaluar si tuvo efectos en las relaciones estimadas.
} 
en las fechas identificadas, pero no establece si en los subperiodos (regímenes) resultantes tales relaciones son o no estadísticamente significativas. Es decir, las relaciones pueden cambiar de signo o perder o ganar significancia estadística. Entonces, para complementar el análisis, se estimaron los coeficientes correspondientes. Las estimaciones se muestran en el cuadro 4.

En general, en los resultados a nivel nacional se pueden identificar algunos patrones como los reportados previamente en la literatura cuando se usa el indicador del ciclo generado con el filtro CF. En particular, las estimaciones para el gasto total nacional sugieren que efectivamente la política fiscal fue pro-cíclica entre 1980 y finales de los años noventa, pero que a partir de entonces sus efectos no han sido estadísticamente significativos. ${ }^{21}$ A nivel más específico, los resultados indican que la relación del ciclo con el gasto administrativo y el de obra pública es positivo hasta 1998 y 2009, respectivamente. Desde entonces, sin embargo, ha sido negativo en los dos casos desde 2004 y 2010 hasta 2015, respectivamente, lo que indica que se han seguido políticas contra-cíclicas en el primer caso desde que culmina la recesión de inicios de siglo y en el segundo desde el término de la Gran Recesión (Mejía et al., 2017). Si combinamos la evidencia de todos los subperiodos analizados se podría ver este proceso de ajuste como parte de una estrategia de largo plazo más general que busca reducir la participación del Estado en la economía (Moreno y Ros, 2010). Nótese que las estimaciones mostradas en el cuadro 3 implican que este patrón no se aplica para los gastos en transferencias, las cuales tiene una relación positiva, significativa y estable con los dos indicadores del ciclo que aquí consideramos; es decir, este rubro del gasto público es pro-cíclico en todo el periodo de estudio.

21 Llama la atención, aun cuando carece de significancia estadística, la reducción de la magnitud del coeficiente pendiente a medida que pasa el tiempo hasta que se vuelve negativo en el periodo 2010-2015. Este último caso, apoya el argumento de que la política fiscal contra-cíclica seguida durante y después de la Gran Recesión ha sido insuficiente e inefectiva, como afirman Villagómez y Navarro (2010). 


\section{Cuadro 4}

COEFICIENTES ESTIMADOS POR RÉGIMEN PARA LA RELACIÓN ENTRE EL CICLO ECONÓMICO Y LOS COMPONENTES DEL GASTO PÚBLICO DE LOS ESTADOS DEL CENTRO DE MÉXICO

\begin{tabular}{|c|c|c|c|c|c|c|c|}
\hline \multirow{2}{*}{$\begin{array}{l}\text { Componente } \\
\text { de Gasto }\end{array}$} & \multirow{2}{*}{ Entidad } & \multicolumn{3}{|c|}{$\mathrm{CF}$} & \multicolumn{3}{|c|}{ TC } \\
\hline & & Regímenes & Interceptos & C. Pendiente & Regímenes & Interceptos & C. Pendiente \\
\hline \multirow{13}{*}{ Gasto Total } & \multirow{4}{*}{ Nacional } & 1980-1998 & -0.001 & 0.156 & & & \\
\hline & & $1999-2004$ & -0.010 & 0.815 & & & \\
\hline & & $2005-2009$ & 0.013 & 0.402 & & & \\
\hline & & $2010-2015$ & -0.004 & -0.340 & & & \\
\hline & \multirow{2}{*}{$\begin{array}{l}\text { Estado de } \\
\text { México }\end{array}$} & & & & $1980-1986$ & 3.66 & 0.314 \\
\hline & & & & & $1987-2015$ & 1.729 & 0.08 \\
\hline & \multirow{2}{*}{ Morelos } & $1980-2011$ & -0.001 & 0.223 & & & \\
\hline & & $2012-2015$ & 0.010 & -0.152 & & & \\
\hline & \multirow{3}{*}{ Querétaro } & 1980-1987 & -0.001 & 0.464 & \multirow{3}{*}{$\begin{array}{l}1980-1987 \\
1988-2015\end{array}$} & \multirow{3}{*}{$\begin{array}{c}-0.067 \\
5.93\end{array}$} & \multirow{3}{*}{$\begin{array}{r}0.428 \\
-0.132\end{array}$} \\
\hline & & 1988-1993 & 0.001 & 0.107 & & & \\
\hline & & $1994-2015$ & 0.000 & -0.095 & & & \\
\hline & \multirow{2}{*}{ Tlaxcala } & & & & $1980-1986$ & 7.01 & 0.193 \\
\hline & & & & & $1987-2015$ & 0.946 & 0.084 \\
\hline \multirow{15}{*}{$\begin{array}{c}\text { Gasto } \\
\text { Administrativo }\end{array}$} & \multirow{3}{*}{ Nacional } & $1980-1998$ & -0.002 & 0.288 & & & \\
\hline & & 1999-2003 & 0.010 & 0.719 & & & \\
\hline & & 2004-2015 & 0.001 & -0.500 & & & \\
\hline & Ciudad de & & & & 1980-1987 & -2.597 & 0.061 \\
\hline & México & & & & $1988-2015$ & 2.327 & 0.144 \\
\hline & \multirow{2}{*}{ Guanajuato } & $1980-1986$ & 0.006 & -1.048 & & & \\
\hline & & $1987-2015$ & -0.001 & 0.003 & & & \\
\hline & \multirow{2}{*}{$\begin{array}{l}\text { Estado de } \\
\text { México }\end{array}$} & $1980-1998$ & -0.002 & 0.126 & & & \\
\hline & & $1999-2015$ & 0.000 & -0.449 & & & \\
\hline & \multirow{2}{*}{ Morelos } & 1980-1986 & 0.004 & 0.318 & & & \\
\hline & & $1987-2015$ & -0.001 & -0.045 & & & \\
\hline & \multirow{2}{*}{ Puebla } & $1980-1986$ & 0.000 & 0.424 & 1980-1987 & 2.35 & 0.337 \\
\hline & & $1987-2015$ & 0.000 & 0.088 & $1988-2015$ & 2.46 & 0.069 \\
\hline & \multirow{2}{*}{ Tlaxcala } & & & & $1980-1986$ & 7.959 & 0.262 \\
\hline & & & & & $1987-2015$ & 1.834 & -0.035 \\
\hline \multirow{14}{*}{ Transferencias } & \multirow{4}{*}{$\begin{array}{l}\text { Ciudad de } \\
\text { México }\end{array}$} & & & & $1980-1986$ & 2.06 & -0.095 \\
\hline & & $1980-1985$ & -0.006 & -0.084 & $1987-1996$ & 0.74 & 0.205 \\
\hline & & $1986-2015$ & 0.000 & 0.070 & $1997-2001$ & 4.735 & -0.002 \\
\hline & & & & & $2002-2015$ & 2.454 & -0.069 \\
\hline & \multirow{2}{*}{ Hidalgo } & $1980-1986$ & 0.010 & 0.681 & $1980-1986$ & -2.612 & 0.709 \\
\hline & & $1987-2015$ & 0.000 & 0.010 & $1988-2015$ & 2.804 & -0.005 \\
\hline & Estado de & $1980-2000$ & -0.001 & -0.024 & & & \\
\hline & México & 2001-2017 & -0.001 & 0.152 & & & \\
\hline & Morelos & $1980-1985$ & -0.008 & 0.421 & $1980-1986$ & -4.533 & 0.462 \\
\hline & & $1986-2015$ & -0.001 & 0.065 & $1987-2015$ & 2.86 & 0.01 \\
\hline & Puebla & $1980-2012$ & -0.002 & 0.076 & & & \\
\hline & & $2013-2015$ & 0.02 & -0.298 & & & \\
\hline & Querétaro & $1980-1987$ & 0.013 & -1.345 & 1980-1987 & 15.165 & -1.578 \\
\hline & & $1988-2015$ & 0 & -0.022 & $1988-2015$ & 5.061 & 0.01 \\
\hline
\end{tabular}




\section{Cuadro 4 (Continuación)}

COEFICIENTES ESTIMADOS POR RÉGIMEN PARA LA RELACIÓN ENTRE EL CICLO ECONÓMICO Y LOS COMPONENTES DEL GASTO PÚBLICO DE LOS ESTADOS DEL CENTRO DE MÉXICO

\begin{tabular}{|c|c|c|c|c|c|c|c|}
\hline \multirow{2}{*}{$\begin{array}{c}\text { Componente } \\
\text { de Gasto }\end{array}$} & \multirow{2}{*}{ Entidad } & \multicolumn{3}{|c|}{ CF } & \multicolumn{3}{|c|}{ TC } \\
\hline & & Regímenes & Interceptos & C. Pendiente & Regímenes & Interceptos & C. Pendiente \\
\hline \multirow{5}{*}{ Transferencias } & \multirow{5}{*}{ Tlaxcala } & $1980-1986$ & 0.015 & 0.4 & 1980-1987 & -10.457 & 0.569 \\
\hline & & $1987-1992$ & -0.013 & -0.096 & 1988-1996 & -0.205 & -0.006 \\
\hline & & 1993-1997 & -0.013 & 0.027 & 1997-2001 & 7.123 & -0.012 \\
\hline & & $1998-2002$ & 0.01 & -0.027 & 2002-2015 & 0.26 & 0.281 \\
\hline & & 2003-2015 & 0 & 0.192 & & & \\
\hline \multirow{18}{*}{ Obra Pública } & \multirow{2}{*}{ Nacional } & 1980-2009 & 0.002 & 0.111 & & & \\
\hline & & 2010-2015 & -0.003 & -0.095 & & & \\
\hline & \multirow{3}{*}{$\begin{array}{l}\text { Ciudad de } \\
\text { México }\end{array}$} & 1980-1985 & 0.001 & 0.264 & 1980-1987 & -0.952 & 0.203 \\
\hline & & $1986-2015$ & 0.001 & -0.027 & 1988-1993 & 5.009 & 0.045 \\
\hline & & & & & 1994-2015 & 2.126 & -0.023 \\
\hline & \multirow{2}{*}{ Hidalgo } & & & & $1980-1988$ & 2.807 & -0.15 \\
\hline & & & & & $1989-2015$ & 2.556 & 0.028 \\
\hline & \multirow{4}{*}{$\begin{array}{l}\text { Estado de } \\
\text { México }\end{array}$} & & & & 1980-1986 & 4.188 & 0.281 \\
\hline & & & & & 1987-1996 & 0.271 & 0.118 \\
\hline & & & & & $1997-2001$ & 6.571 & 0.018 \\
\hline & & & & & 2002-2015 & 2.665 & 0.068 \\
\hline & \multirow{3}{*}{ Morelos } & $1980-1986$ & 0.003 & 0.359 & 1980-1987 & -6.439 & 0.314 \\
\hline & & $1987-2015$ & -0.001 & -0.019 & 1988-1993 & 7.191 & 0.056 \\
\hline & & & & & 1994-2015 & 2.5 & -0.043 \\
\hline & \multirow{2}{*}{ Querétaro } & $1980-2011$ & 0 & -0.052 & & & \\
\hline & & 2012-2015 & 0.015 & 0.036 & & & \\
\hline & \multirow{2}{*}{ Tlaxcala } & & & & 1980-1986 & 9.64 & -0.06 \\
\hline & & & & & $1987-2015$ & 1.737 & -0.012 \\
\hline
\end{tabular}

Valores significativos al $5 \%$ en negritas y significativos al $10 \%$ en cursivas.

Fuente: estimaciones propias.

Las estimaciones de los coeficientes para los diferentes regímenes a nivel estatal ofrecen resultados menos claros y robustos, aunque se puede identificar algunos patrones interesantes. Primero, la mayor parte de los regímenes con coeficientes significativos se vinculan con quiebres que se presentan en los años ochenta, lo que, como se mencionó, puede explicarse por el cambio en la intensidad de las políticas de estabilización que hasta 1987 se basó en fuertes recortes del gasto público en las fases recesivas. Aunque después se mantuvo una política esencialmente similar, al menos hasta inicios de la década pasada, los ajustes fueron más moderados. Segundo, como consecuencia, los coeficientes pendiente positivos reducen su magnitud (y en ocasiones pierden su significancia estadística) cuando se pasa a regímenes más recientes, como en los casos del gasto total del Estado de México (TC), 
gastos administrativos de Puebla (CF y TC), transferencias de Hidalgo (CF) y de Morelos (CF y TC) y gasto en obra pública del Estado de México (TC) y Morelos (TC), por citar algunos ejemplos. Tercero, el coeficiente pendiente pasa de positivo a negativo en el gasto total de Querétaro (TC), gastos administrativos del Estado de México (TC) y Morelos (TC), transferencias de Hidalgo (TC) y gasto en obra pública de la Ciudad de México (CF). ${ }^{22}$ Tercero, en muy pocos casos el coeficiente pendiente cambia de negativo a positivo, como en los gastos administrativos de Guanajuato (CF), las transferencias del Estado de México (CF) y de Querétaro (TC). Por último, llama la atención la gran cantidad de quiebres estructurales que se presenta en la relación del ciclo con las transferencias en el estado de Tlaxcala y la alternancia de los signos de dicha relación. No obstante, al parecer existe una relación robusta durante los primeros dos y el último régimen, tanto en términos de signo de la relación como de subperiodos de tiempo.

\section{CONCLUSIONES}

Se analizó la relación entre algunos componentes del gasto público y los ciclos económicos de la economía nacional y de los estados del centro de México, una región especialmente importante, pero escasamente estudiada en la literatura, para el periodo 1980-2015. El análisis se realizó a partir de la prueba de Bai y Perron (1998) para evaluar la posibilidad de que se presenten múltiples cambios estructurales en dicha relación.

Los resultados sugieren la presencia de cambios estructurales en las diferentes relaciones durante la segunda mitad de los años ochenta y el transcurso de los noventa, cuando la economía mexicana experimenta un alto grado de inestabilidad y se recurre a vastos recortes de gasto público como estrategia estabilizadora, mientras que otros se presentan en torno a las recesiones de 2001-2003, cuando se aplica una política convencional de recortes en el gasto, aun cuando la recesión era de naturaleza diferente y de una magnitud moderada, y 2008-2009, cuando la política fiscal siguió por primera vez una lógica contra-cíclica. Los cambios identificados en torno a 1998 podrían vincularse a la modifica-

22 Algo similar ocurre con el coeficiente negativo de la relación del ciclo con las transferencias en el estado de Querétaro (CF). 
ción de la Ley de Coordinación Fiscal de ese año que se concretó en la creación del rubro de aportaciones a los estados (Ramo 33).

Las estimaciones de los coeficientes de los regímenes implicados por los cambios estructurales identificados sugieren patrones interesantes a nivel nacional. Los resultados del gasto total nacional indican que efectivamente la política fiscal fue pro-cíclica entre 1980 y finales de la década de los años noventa cuando el análisis se hace en la frecuencia del ciclo (filtro CF). Interesantemente, a partir de entonces la relación con el ciclo deja de ser estadísticamente significativa. Más aun, para el periodo 2010-2015 se estima un signo negativo, el cual resulta no significativo, lo que apoya la hipótesis de que la política contra-cíclica seguida desde entonces ha sido ineficaz. A nivel desagregado, sin embargo, hay evidencia de tal cambio de política, aunque para periodos diferentes, en los casos del gasto administrativo y el de obra pública. Las transferencias, por su parte, siempre han sido pro-cíclicas, sugiriendo una alta sensibilidad a la disponibilidad de recursos.

A nivel estatal los resultados son menos robustos, aunque se pueden identificar algunos patrones interesantes. Primero, los cambios estructurales se presentan aproximadamente en las mismas fechas en que ocurren a nivel nacional, aunque en una proporción importante de los estados corresponden a la década de los años ochenta. Segundo, los quiebres no son robustos al método de eliminación de tendencia, lo que podría explicarse por el hecho de que el filtro CF genera un indicador correspondiente a la frecuencia del ciclo de crecimiento, en tanto que las TC se pueden vincular a la idea del ciclo clásico, lo que hace que los resultados sean complementarios más que excluyentes. Tercero, los coeficientes-pendiente estimados reducen su magnitud y en ocasiones pierden su significancia estadística e incluso se tornan negativos. Cuarto, al igual que a nivel nacional, se puede argumentar que el manejo del gasto público en múltiples estados ha pasado en diferentes momentos de pro-cíclico a a-cíclico o contra-cíclico, lo que ha estado determinado por el activo papel de la política fiscal en la estabilización de la economía nacional en la década de los años ochenta y noventa y por el modesto impacto que ha tenido en los años recientes.

En general, se pueden obtener algunas implicaciones interesantes a partir de nuestros resultados. Primero, los patrones identificados reflejan la alta dependencia del gasto que ejercen los estados de la disponibilidad de recursos y de la orientación de la política fiscal a nivel federal. 
Segundo, la presencia de múltiples cambios estructurales en algunas relaciones podría reflejar la elevada inestabilidad en el manejo del gasto producto de la alta discrecionalidad en su manejo. Tercero, la no significancia estadística de los coeficientes estimados sugiere que el gasto es realmente infectivo en la estabilización de los ciclos estatales o que se usa con criterios diferentes, entre los que se podría contar su uso con fines político-electorales.

En cualquier caso, resulta evidente la necesidad de profundizar en el análisis de la dinámica económica de los estados y en el de los efectos de la política económica a nivel subnacional con la finalidad de proveer a los gobiernos estatales de herramientas que puedan usarse para amortiguar las fluctuaciones idiosincráticas, dejadas de lados por políticas nacionales.

\section{ANEXO. INFORMACIÓN ESTADÍSTICA}

La base de datos del gasto público y sus componentes se integró a partir de la información presentada en los anuarios El ingreso y el gasto público en México, varios números (1980-1989) y el Sistema Estatal y Municipal de Base de Datos (SIMBAD) del Instituto Nacional de Estadística, Geografía e Informática (1990-2015); los detalles se presentan en la bibliografía. Las variables consideradas y sus definiciones son las siguientes: gasto total se refiere a la suma de los gastos erogados por los estados, y en el caso nacional corresponde a los ejercidos por los 32 estados del país, por lo que excluye los gastos del gobierno federal; el gasto administrativo se asocia al ejercido en el desempeño de las funciones administrativas de los estados y en la prestación de los servicios públicos que realizan; el gasto en obra pública corresponde al monto destinado a la construcción de obras públicas o a la adquisición de inmuebles y otras inversiones públicas y, por último, las transferencias son las erogaciones originadas por terceros, a quienes se les transfiere para dichos fines los subsidios o subvenciones, ayudas o donativos, aportaciones o cooperaciones e intereses de la deuda pública (INEGI, 1986; INEGI 1993). Debido a que en 2002 cambia la clasificación del gasto según su concepto, desglosándose nuevos rubros, las series se homogeneizaron agregando los siguientes componentes: en gastos administrativos se sumaron servicios personales, materiales y suministros y servicios generales; en obras públicas, adquisición de 
bienes muebles e inmuebles y obras públicas y acciones sociales; y en transferencias, subsidios, transferencias y ayudas y recursos federales y estímulos a municipios.

El PIB estatal se obtuvo de las estimaciones realizadas por GermanSoto (2005), se encadenó en 2003 con datos obtenidos del SIMBAD. Las series nominales se deflactaron con el Índice de Precios Implícito (IPI) del PIB estatal, base 2008.

Para evitar que las observaciones anómalas (definidas como aquéllas cuyos valores rebasan \pm 3 desviaciones estándar) afectaran el análisis se les reemplazó por el promedio del dato anterior y posterior, como sugieren Artis et al. (1997).

\section{Bibliografía}

Aizenman, Joshua y Yothin Jinjarak (2011). The role of fiscal policy in response to the financial crisis, United Nations, New York. $<$ http://csf.rrojasdatabank.info/wesp2011paper_aizenman.pdf > [30 de marzo de 2016].

Alesina, Alberto; Filipe R. Campante y Guido Tabellini (2008). "Why is fiscal policy often procyclical?", Journal of the European Economic Association, 5(6), Oxford University Press, Oxford, pp. 1006-1036.

Alesina, Alberto (2012). "Fiscal Policy after the Great Recession", Atlantic Economic Journal, 40(4), Atlantic Economic Society, Nueva Jersey, pp. 429-435.

Artis, Michael J.; Zenon G. Kontolemis y Denise R. Osborn (1997). "Business cycles for G7 and European Countries", The Journal of Business, 70(2), The University of Chicago Press, Chicago, pp. 249-279.

Arechederra, Fernando K. y Carlos M. Urzúa (2017). "La Ley de Coordinación Fiscal en México: una crítica aritmética", Sobre México. Temas de Economía, 3(1), Universidad Iberoamericana, México, pp. 4-13.

Bai, Jushan y Pierre Perron (2003). "Critical values for multiple structural change tests", Econometrics Journal, 6(1), Royal Economic Society, Nueva Jersey, pp. 72-78.

Bai, Jushan y Pierre Perron (1998). "Estimating and testing linear models with multiple structural changes", Econometrica, 66(1), The Econometric Society, New York, pp. 47-78,

Banco de México (2009). Informe Anual. México.

Banco de México (2011). Reporte sobre las economías regionales. México.

Blanchard, Olivier; Giovanni Dell'Ariccia y Paolo Mauro (2010). "Rethinking macroeconomic policy", Journal of Money, Credit and Banking, 42(1), 
Wiley Online Library, pp. 199-215.

Burns, Arthur F. y Wesley C. Mitchell (1946). Measuring business cycles. National Bureau of Economic Research Books, Nueva York.

Burnside, Craig y Yuliya Meshcheryakova (2005). "Mexico: a case study of procyclical fiscal policy", en Fiscal sustainability in the theory and practice, pp. 133-174. The World Bank, Washington.

Fabrizio Carmignani y James Laurenceson (2013). "Provincial business cycles and fiscal policy in China", Economics of Transition, 21(2), Wiley Online Library, Londres, pp. 323-340.

Cámara de Diputados (1998). Ley de Coordinación Fiscal. México. <http:// www.diputados.gob.mx/LeyesBiblio/ref/lcf.htm> [2 de febrero de 2018].

Cámara de Diputados (2007). Ley de Coordinación Fiscal. México. <http:// www.diputados.gob.mx/LeyesBiblio/ref/lcf.htm> [2 de febrero de 2018].

Christiano, Lawrence J. y Terry Fitzgerald (2003). "The band pass filter", International Economic Review, 44(2), Wiley Online Library, Pennsylvania, pp.435-465.

Cuadra, Gabriel (2008). Hechos estilizados del ciclo económico en México, Documento de investigación no. 2008-14, Banco de México, México.

Eggertsson, Gauti B. (2011). "What fiscal policy is effective at zero interest rates?" en Daron Acemoglu y Michael Woodford (eds.) NBER Macroeconomics Annual 2010, University of Chicago Press, Chicago, pp. 59-112.

Erquizio, Alfredo (2007). "Identificación de los ciclos económicos en México, 1949-2006", Problemas del desarrollo, 38(150), Instituto de Investigaciones Económicas, UNAM, México, pp. 235-250.

Esquivel, Gerardo (2010). De la inestabilidad macroeconómica al estancamiento estabilizador: el papel del diseño y la conducción de la política económica, en Nora Lustig (coord.), Los grandes problemas de México, Vol. 1, Crecimiento económico y equidad, Colegio de México, México, pp.35-77.

Fonseca, Felipe de Jesús; Juan Carlos Chávez y Ricardo Rodríguez (2013). "Estabilización de transferencias federales: Una propuesta para los estados mexicanos basada en el ciclo económico", Economía Mexicana. Nueva época, XXII (1), CIDE, México, pp. 151-175.

Gabriele Galati y Richhild Moessner (2013). "Macroprudential policy - a literature review", Journal of Economic Surveys, 27(5), Wiley Online Library, pp. 846-878.

German-Soto, Vicente (2005). "Generación del Producto Interno Bruto mexicano por entidad federativa, 1940-1992", Trimestre Económico, LXXII (3), Fondo de Cultura Económica, México, pp. 617-653.

Greenwald, Bruce y Joseph E. Stiglitz (1987), "Keynesian, New Keynesian and New Classical Economics", Oxford Economic Papers, 39(1), Oxford University Press, Oxford, pp. 119-133. 
Hori, Hajime (2017). Business cycle dynamics and stabilization policies: a keynesian approach, Springer, Singapore.

Instituto Nacional de Estadística y Geografía (2017). Sistema Estatal y Municipal de Bases de Datos (SIMBAD), 1990-2015. <http://sc.inegi.org.mx/ cobdem $>$ [6 de septiembre de 2017].

Instituto Nacional de Estadística y Geografía (INEGI) (2014). Estadísticas de Finanzas Públicas Estatales y Municipales de México, 1980-1989. <http:// www.inegi.org.mx/sistemas/bie $>$ [26 de noviembre de 2014].

Instituto Nacional de Estadística y Geografía (INEGI) (1986). El ingreso y el gasto público en México. México.

Instituto Nacional de Estadística y Geografía (INEGI) (1993). El ingreso y el gasto público en México. México.

Jones, C. I (2014). Macroeconomics, Norton \& Company, Washington.

Knoop, Todd A. (2015). Business cycle economics: understanding recessions and depressions from boom to bust, Praeger, Santa Barbara.

Kydland, Finn E. y Edward C. Prescott (1990). "Business cycle: real facts and a monetary myth", Federal Reserve Bank of Minneapolis Quarterly Review, Federal Reserve Bank of Minneapolis, Minneapolis 14(2), pp. 3-18.

Kydland, Finn E. y Edward C. Prescott (1982). "Time to build and aggregate fluctuations", Econometrica, 50(6), The Econometric Society, New York, pp. 1345-1370.

Loría, Eduardo y Emmanuel Salas (2014). "Mexico and the United States: cycle synchronization, 1980.1-2013.4”, Ensayos Revista de Economía, XXXIV(1), Facultad de Economía UANL, Monterrey, pp. 75-102.

Lucas, Robert E. (1977). "Understanding business cycles”, Carnegie-Rochester Conference on Public Policy, 5(1), Bradley Policy Research Center, Nueva York, pp. 7-29.

Mandujano, Nicolás (2010). Federalismo fiscal en México. Una propuesta para fortalecer la hacienda pública estatal, UNAM, México.

Mankiw, Gregory N. (1989). "Real business cycles: A new keynesian perspective", The Journal of Economic Perspectives, 3(3), American Economic Association, Washington, pp. 79-90.

Mejía, Pablo (2003). "Regularidades empíricas en los ciclos económicos de México: producción, inversión, inflación y balanza comercial", Economía mexicana. Nueva época, XII(2), CIDE, México, pp.231-274.

Mejía, Pablo; Miguel Ángel Díaz y Reyna Vergara (2017). "Recesiones en México en los albores del Siglo XXI”, Problemas del Desarrollo, 48(189), Instituto de Investigaciones Económicas, UNAM, México, pp. 57-84.

Mejía, Pablo; J. Alonso Martínez y W. Liliana Rendón (2005). “Ciclos económicos industriales clásicos en México”, Investigación económica, LXIV(254), UNAM, México, pp. 91-124. 
Miyazaki, Tomomi (2013). Fiscal policy and regional business cycle fluctuations in Japan, Discussion Paper Series A no. 583, Hitotsubashi University, Japón.

Moreno, J. C. y Jaime Ros (2010). Desarrollo y crecimiento en la economía mexicana. Una perspectiva histórica. Fondo de Cultura Económica, México.

Pérez, P. J.; Denise R. Osborn y Marianne Sensier (2007). "Business cycle affiliations in the context of European integration", Applied Economics, 39 (2), Taylor \& Francis, Didcot, pp. 199-214.

Plosser, C. I (1989). "Understanding real business cycles", The Journal of Economics Perspectives, 3(3), American Economic Association, Washington, pp. 51-78.

Rebelo, Sergio (2005). "Real business cycle models: past, present and future", Scandinavian Journal of Economics, 107(2), Wiley Online Library, pp. 217

Reyes, Marlen y Pablo Mejía (2012). “Co-movimiento de los componentes del gasto público y el ciclo económico en México, 1980-2007”, Explanans, 1(2), Campus Universitario Siglo XXI, México, pp. 53-88.

Rodden, Jonathan y Erik Wibbels (2010). "Fiscal decentralization and the business cycle: an empirical study of seven federations", Economics and politics, 22(1), Wiley Online Library pp. 37-67.

Savva, Christos; Kyriakos C. Neanidis y Denise R. Osborn (2010). "Business cycle synchronization of the Euro Area with the new and negotiating member countries", International Journal of Finance and Economics, 15(3), Washington, pp. 288-306.

Sorensen, Peter B. y Hans J. Whitta-Jacobsen (2010). Introducing advanced macroeconomics: growth and business cycles, McGraw-Hill, Edimburgo.

Spiegel, Shari (2007). Políticas macroeconómicas y de crecimiento, Guías de orientación de políticas públicas, United Nations Department of Economic and Social Affairs, Nueva York.

Stadler, George W (1994). "Real business cycles", Journal of Economic Literature, 32(4), American Economic Association, Washington, pp.17501783.

Stock, James H. y Mark W. Watson (2003). "How did leading indicator forecasts perform during the 2001 recession?", Economic Quarterly, 89(3), Federal Reserve Bank of Richmond, Virginia pp.71-90.

Valdivia, D. y Yujra, P (2009). Identificación de ciclos económicos en Bolivia 1970-2008, Banco Central de Bolivia, La Paz.

Velázquez, César (2006). "Determinantes del gasto estatal en México", Gestión y Política Pública, XV(1), CIDE, México, pp. 83-109. 
Venegas, Francisco y Abigail Rodríguez (2009). "Exogeneidad de la rigidez salarial en la Nueva Economía Keynesiana", Análisis económico, 24(55), Universidad Autónoma Metropolitana, México, pp. 303-326.

Villagómez, Alejandro y Luis Navarro (2010). Política fiscal contracíclica en México durante la crisis reciente: un análisis preliminar, Documento de Trabajo DTE 475, Centro de Investigación y Docencia Económica, México.

Williamson, John (2009). "A Short History of the Washington Consensus", Law and Business Review of the Americas, 15(1), Southern Methodist University, Texas, pp. 7-23. 